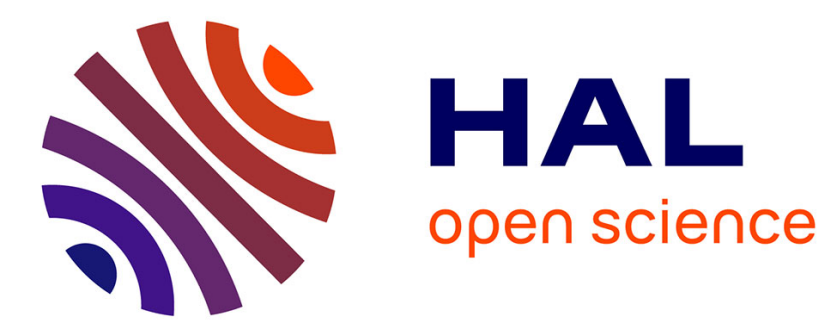

\title{
About an inverse problem for a free boundary compressible problem in hydrodynamic lubrication
}

\author{
K. Ait Hadi, Guy Bayada, M. El Alaoui Talibi
}

\section{To cite this version:}

K. Ait Hadi, Guy Bayada, M. El Alaoui Talibi. About an inverse problem for a free boundary compressible problem in hydrodynamic lubrication. Journal of Inverse and Ill-posed Problems, 2015. hal-00822388

\section{HAL Id: hal-00822388 \\ https://hal.science/hal-00822388}

Submitted on 15 May 2013

HAL is a multi-disciplinary open access archive for the deposit and dissemination of scientific research documents, whether they are published or not. The documents may come from teaching and research institutions in France or abroad, or from public or private research centers.
L'archive ouverte pluridisciplinaire HAL, est destinée au dépôt et à la diffusion de documents scientifiques de niveau recherche, publiés ou non, émanant des établissements d'enseignement et de recherche français ou étrangers, des laboratoires publics ou privés. 


\title{
About an inverse problem for a free boundary compressible problem in hydrodynamic lubrication
}

\author{
K. Ait Hadi - G. Bayada • \\ M. El Alaoui Talibi
}

Received: date / Accepted: date

\begin{abstract}
In this paper an inverse problem is considered for a non coercive partial differential equation, issued from a mass conservation cavitation model for a slightly compressible fluid. The cavitation phenomenon and compressibility take place and are described by the Elrod model. The existence of an optimal solution is proven. Optimality conditions are derived and some numerical results are given.
\end{abstract}

Keywords Inverse problem · Elrod model · optimality system · numerical results.

Mathematics Subject Classification (2000) MCS 35J70 - MCS 35Q35 . MCS 49K20 - MCS 65N21 · MCS 65N30.

\section{Introduction}

The present work comes within the scope of inverse problems in hydrodynamic lubrication. Numerous works are based upon the computation of the solution of various forms of the Reynolds partial differential equation established in 1886. This equation enables one to compute the hydrodynamic pressure $(P)$

Khalid Ait Hadi

Université Cadi Ayyad, Laboratoire LIBMA de Mathématiques Appliquées Faculté des Sciences Semlalia, Département de Mathématiques, BP 2390, Marrakech Morocco.

E-mail: k.aithadi@ucam.ac.ma

Guy Bayada

Université de Lyon, INSA, Institut Camille Jordan, UMR CNRS 5208, Bat . Leonard de Vinci Math 69621 Villeurbanne Cedex France.

E-mail: guy.bayada@insa-lyon.fr

Corresponding author: Mohamed El Alaoui Talibi

Université Cadi Ayyad, Laboratoire LIBMA de Mathématiques Appliquées Faculté des Sciences Semlalia, Département de Mathématiques, BP 2390, Marrakech Morocco.

E-mail: elalaoui@uca.ma 
in a lubricated device, from data like the velocities distribution on the surfaces surrounding the thin film flow, the gap between these surfaces(or equivalently the shape of one of these surfaces) and the rheological characteristics of the fluids. However, due to severe operational conditions(the gap between the surfaces can be of some micrometers only and the relative velocity of the surfaces some meters/second), some of these data are not really well known.

This is the case for example of the surfaces which are deformed from an initial known shape by the hydrodynamic pressure inside the fluid. Experimentally, the knowledge of this pressure $(P)$ can now be obtained with a good precision. It becomes then possible to find the real shape $(h)$ of the surfaces by solving an inverse problem with Reynolds equation as state equation. Such information is important as if the related gap is too small, it means that some contact is possible between the two surfaces thus inducing wear and possible failure of the device.

Two other very similar problems in the lubrication field can be mentioned. The first one is to find the gap $(h)$ such that the pressure $(P)$ is the greatest possible. In some specific situation the solution is the Rayleigh-step bearing [31] in which the optimum gap $h$ is a discontinuous function. More recently, generalized Reynolds equation for heterogeneous slip/no slip engineered surfaces have been proposed [9]. As a consequence, a no-slip condition for the velocity is valid on some part of the fluid boundary and slip occurs on the other part. Due to this non homogeneity, the resulting Reynolds equation contains discontinuous coefficients. Finding the best location of the slip/no-slip regions is mathematically close to the previous optimization problem.

Another difficulty must be considered in these aforementioned identificationoptimization problems. It is due to cavitation, the well-known phenomenon in fluid mechanics when fluid is no longer homogeneous and takes some diphasic aspect with the appearance of air bubbles. This phenomenon occurs very often in lubrication and cannot be ignored, especially as the gap $(h)$ is not constant. Taking cavitation into account implies considering a new non linear operator as state equation instead of the classical Reynolds equation. Various models exist in the mechanical literature. The most common one is based upon a variational inequality for the pressure [27]. However, as it is not a mass preserving model, it is often replaced by the Jacobsson Floberg Olssen (JFO) model, in which a new variable $\theta$ is introduced. This variable describes the local proportion of fluid (or saturation) considering the presence of air bubbles in the flow [25]. In a widely referenced paper [20], it has been pointed out that the (JFO) model is equivalent to a free boundary complementary problem with two unknowns, pressure $P$ and saturation $\theta$ with

$$
P \geq 0,0 \leq \theta \leq 1, P(1-\theta)=0
$$

Existence and uniqueness aspects of such models have been mathematically studied in a lot of papers [2] . However another aspect of the Elrod-Adams paper has been less studied in the mathematical literature: authors try to recover the somewhat heuristic JFO model by introducing a small compressibility in the classical (non cavitated) Reynolds equation. They show that the JFO 
model can be obtained as the limit of this compressible model and propose some numerical procedures to solve it. Although being somewhat heuristic, this result has been supported by recent rigorous mathematic results [14] for the asymptotic thin film compressible Navier Stokes system. The interest of the more physical compressible Elrod-Adams model is that there is only one unknown (the density of lubricant) instead of two $(P$ and $\theta)$ in the JFO model. The price to pay is that the state equation is a non linear degenerate partial differential equation. The importance of the choice of a model of cavitation in the simulation of lubrication devices has often been mentioned $[9,4]$.

Although these inverse-optimization problems are of permanent interest in the mechanical literature $[3,12,15,17,19,21,29]$, few mathematical works exist. One of the first attempts [29] is devoted to the optimum Rayleight step bearing. In [30] the identification procedure for a gap with known pressure is studied. However, in these works cavitation is not considered. In [8], variational inequality is chosen to model the cavitation and optimality problems are studied by way of penalization. The Elrod-Adams model is considered in [19] using some regularizations which enables one to gain optimality conditions. The present study addresses the identification process of the gap for a given pressure and takes the cavitation into account by way of the slightly compressible model proposed by Elrod-Adams. This model has also the advantage of preserving the mass flow.

This problem presents many mathematical difficulties essentially bound to the complexity of the criterion considered as a function of the thickness. It is not even a locally Lipschitz function. We are lead to consider a more general functional framework which is the space of the functions of bounded variation. To deal with these difficulties a double regularization is introduced by approaching the degenerate part of the state equation with a particular sequence of monotonous and continuously differentiable functions. Thus a sequence of regularized control problems can be obtained.

The paper is organized as follows. Section 2 is devoted to the physical background, the statement of the problem and the proof of the existence of a control. In section 3, we formulate the regularized control problem for which an existence result is proved. Optimality conditions are given in section 4, and some a priori estimates are obtained, so allowing us to pass to the limit. The optimality system for the initial problem is derived in the one-dimensional case. In the last section some numerical results show that the optimality system associated to an inverse algorithm is efficient.

Finally let us mention that from a mathematical aspect, the problem here studied is very close to the one of the "dam problem" [13] with unsaturated porous media or multiple fluid saturated porous media. In this case, the unknowns to be determined are the value of the porosity parameters of the soil [18] and the measured data is the hydraulic pressure. 


\section{Formulation of the problem and existence of control}

2.1 Description of the physical problem (Elrod-Adams model)

Let $\Omega$ the square $] 0,1[\times] 0,1\left[\right.$ of the $(x, y)$ plane, $\Gamma_{0}=\{(x, y) \in \partial \Omega, x=0\}$, and $\Gamma=\partial \Omega \backslash \Gamma_{0}$. The lubricant is assumed to be contained in a three dimensional volume between the lower flat surface $\Omega$ with horizontal velocity $\vec{U}$ and an upper one describe by $z=h(x, y)$ at rest. If $h$ is small, the pressure is known to be independent from $z$ and to obey the following Reynolds equation defined on $\Omega$ [23].

$$
\operatorname{div}\left(\frac{\rho}{12 \eta} h^{3} \nabla P\right)=\operatorname{div}\left(\frac{h \vec{U} \rho}{2}\right) \text { on } \Omega,
$$

where $P(x, y)$ is the pressure of the lubricant, $h(x, y)$ is the film thickness, $\rho$ is the density of lubricant, $\eta$ is the viscosity, $\vec{U}$ is the relative velocity of the surfaces of the mechanism in which lubricant takes place.

For a slightly compressible fluid like water or oil, the law linking $P$ and $\rho$ is usually described by introducing the bulk number $\beta$ with: see [25]

$$
P=\beta \log (\rho), \rho>1, P>0,
$$

Taking into account the possible existence of a cavitation area where $P$ is zero and $\rho \leq 1$ lead to generalize $(2)$ in

$$
P=\beta \log \left((\rho-1)^{+}+1\right), \rho \geq 0, \beta>0 .
$$

in which $f^{+}=\sup (f, 0)$. Rewriting (1) in term of $\rho$, we gain by differentiating in the distributional sense

$$
\operatorname{div}\left(\beta \frac{h^{3}}{12 \eta} \nabla(\rho-1)^{+}-\rho \frac{h \vec{U}}{2}\right)=0
$$

Introducing $u=\beta(\rho-1)$ as the primary unknown, equation (3) becomes

$$
\operatorname{div}\left(\frac{h^{3}}{12 \eta} \nabla u^{+}-\left(1+\frac{1}{\beta} u\right) \frac{h \vec{U}}{2}\right)=0 .
$$

In the sequel, we will consider more precisely the case of a journal bearing (see Figure 1) with a supply line located at $x=0$. It is a usual lubrication device in which a known quantity of fluid $\Theta_{0}$ is supplied through $\Gamma_{0}=\{(x, y) \in \partial \Omega, x=0,0<y<1\}$ while the pressure is assumed to be known on $\Gamma=\partial \Omega \backslash \Gamma_{0}$ and equal to zero (the atmospheric pressure).

For simplicity sake we assume $\eta=\frac{1}{6}, \vec{U}==\left(\begin{array}{l}1 \\ 0\end{array}\right)$ so that the weak formulation of the state problem reads :

$\left(\mathcal{P}_{h}\right)\left\{\begin{array}{l}\text { Find } u \in L^{2}(\Omega) \text { verifying } u^{+} \in V,\left(1+\frac{1}{\beta} u\right) \geq 0 \\ \int_{\Omega} h^{3} \nabla u^{+} \cdot \nabla \phi d x d y-\int_{\Omega} h\left(1+\frac{1}{\beta} u\right) \frac{\partial \phi}{\partial x} d x d y=\int_{\Gamma_{0}} \Theta_{0} \phi d y \quad \forall \phi \in V \\ \Theta_{0} \in L^{\infty}\left(\Gamma_{0}\right), \Theta_{0} \geq 0\end{array}\right.$ 
with $V:=\left\{\varphi \in H^{1}(\Omega), \varphi_{/ \Gamma}=0\right\}$.
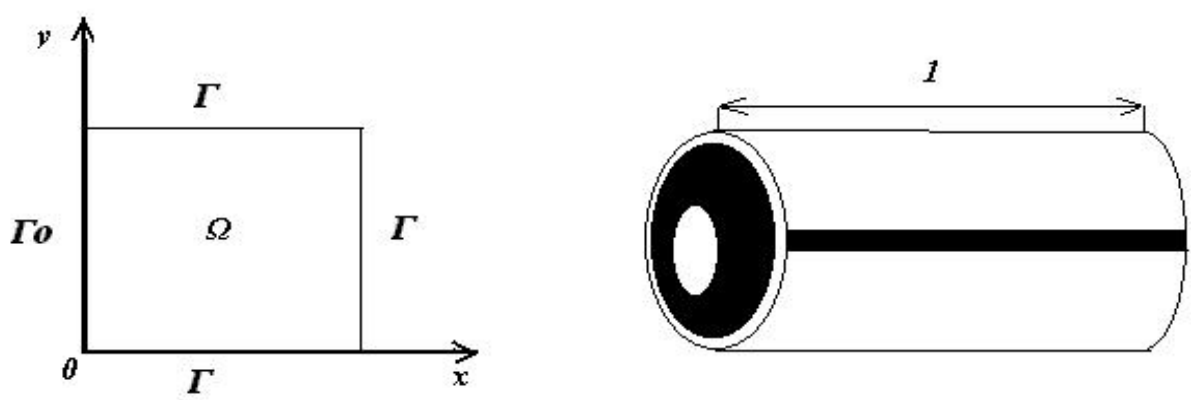

Figure 1: Geometry of a cylindrical journal bearing.

For a given pressure $P_{d}$ in $L^{2}(\Omega)$, we define the following optimal control problem that will be studied in the sequel

$(\mathcal{M})\left\{\begin{array}{l}\operatorname{Min} J(h)=\frac{1}{2}\left\|P(h)-P_{d}\right\|_{L^{2}(\Omega)}^{2}=\frac{1}{2}\left\|\beta \log \left(1+\frac{1}{\beta} u^{+}(h)\right)-P_{d}\right\|_{L^{2}(\Omega)}^{2} \\ h \in \mathcal{U}_{a d}\end{array}\right.$ in which $u(h)$ denotes the solution of $\left(\mathcal{P}_{h}\right)$ for a given $h . \mathcal{U}_{a d}$ wil be defined later.

\subsection{About the state equation}

Problem $\left(\mathcal{P}_{h}\right)$ has been studied in [1] and existence and uniqueness have been obtained for $h=h(x)$ lipschitz continuous function satisfying the condition

$$
\min _{\{x \in[0,1]\}}\left(h^{3}(x)\right)-\frac{1}{\beta} C_{p}\|h\|_{L^{\infty}(\Omega)}>0,
$$

with $C_{p}$ the constant imbedding of $H_{0}^{1}(\Omega)$ into $L^{2}(\Omega)$.

However, as it was detailed in the introduction, discontinuous function $h(x, y)$ could be solutions of some optimization-identification process. The set $U_{a d}$ in $(\mathcal{M})$ is then defined in a more general way by:

$\mathcal{U}_{a d}=\left\{h \in B V(\Omega) \cap L^{\infty}(\Omega) / 0<a \leq h \leq b\right.$ a.e in $\Omega$ and $\left.\|D h\|(\Omega) \leq C^{*}\right\}$

where $C^{*}$ is a given constant and in which $B V(\Omega)$ denotes the space of functions of bounded variations in $\Omega$ :

$$
B V(\Omega)=\left\{f \in L^{1}(\Omega),\|D f\|(\Omega)<+\infty\right\},
$$


with

$$
\|D f\|(\Omega):=\sup \left\{\int_{\Omega} f \operatorname{div} \varphi d x d y / \varphi \in C_{c}^{1}\left(\Omega, \mathbb{R}^{2}\right) ;|\varphi| \leq 1\right\} .
$$

It is known that $B V(\Omega)$ is a Banach space for the following norm

$$
\|f\|_{B V(\Omega)}:=\|f\|_{L^{1}(\Omega)}+\|D f\|(\Omega) .
$$

Some properties of $B V$ spaces are given in Appendix A [Lemma A3-A4].

A close examination of the proof of existence of the solution of $\left(\mathcal{P}_{h}\right)$ shows that it is valid without any change for $h(x, y)$ in $\mathcal{U}_{a d}$ and not only for $h(x)$ lipschitz continuous so that theorems Th. 2.14 and Th.3.4 in [1] can be generalized as:

Theorem 1 With the assumption

$$
a^{3}>\frac{1}{\beta} C_{p} b .
$$

For any $h \in \mathcal{U}_{\text {ad }}$ and any $\Theta_{0}, \Theta_{0} \in L^{\infty}\left(\Gamma_{0}\right), \Theta_{0} \geq 0$, there exists at least a solution $u$ of $\left(\mathcal{P}_{h}\right)$ such that

$$
\begin{gathered}
\|u\|_{L^{2}(\Omega)} \leq C_{1}, \\
\left\|\nabla u^{+}\right\|_{\left(L^{2}(\Omega)\right)^{2}} \leq C_{2},
\end{gathered}
$$

where $C_{1}$ and $C_{2}$ are two positives constants which don't depend on $\beta$.

Regarding the uniqueness of the solution of the state problem, the lack of regularity of the coefficients cannot be easily overcome and we cannot gain this property.

So, the optimal control problem is slightly modified and defined as a two unknowns problem in which the state equation is considered as a constraint:

$(\tilde{\mathcal{M}})\left\{\begin{array}{l}\operatorname{Min} \tilde{J}(h, u)=\frac{1}{2}\left\|P-P_{d}\right\|_{L^{2}(\Omega)}^{2}=\frac{1}{2}\left\|\beta \log \left(1+\frac{1}{\beta} u^{+}\right)-P_{d}\right\|_{L^{2}(\Omega)}^{2} \\ \text { subject to } \\ h \in \mathcal{U}_{a d}=\left\{h \in B V(\Omega) \cap L^{\infty}(\Omega) / 0<a \leq h \leq b \text { a.e in } \Omega \text { and }\|D h\|(\Omega) \leq C^{*}\right\} \\ \text { and } \\ \left\{\begin{array}{l}u \in L^{2}(\Omega) \text { verifying } u^{+} \in V,\left(1+\frac{1}{\beta} u\right) \geq 0 \\ \int_{\Omega} h^{3} \nabla u^{+} \cdot \nabla \phi d x d y-\int_{\Omega} h\left(1+\frac{1}{\beta} u\right) \frac{\partial \phi}{\partial x} d x d y=\int_{\Gamma_{0}} \Theta_{0} \phi d y \quad \forall \phi \in V\end{array}\right.\end{array}\right.$

Theorem 2 There exists at least one solution $\left(h^{*}, u^{*}\right)$ for the problem $(\tilde{\mathcal{M}})$.

Proof Let $\left(h_{n}, u_{n}\right)_{n \in \mathbb{N}} \subset \mathcal{U}_{a d} \times L^{2}(\Omega)$ be a minimizing sequence for the problem $(\tilde{\mathcal{M}})$. According to the Proposition 7, there exists $h^{*} \in \mathcal{U}_{a d}$ and a subsequence $h_{n_{k}}$ such that $h_{n_{k}}$ tends to $h^{*}$ in $L^{\lambda}(\Omega)$ for any $\lambda \in[1,+\infty[$.

By Theorem 1, $\left\|\nabla u_{n_{k}}^{+}\right\| \leq C_{1}$ and $\left\|u_{n_{k}}\right\| \leq C_{2}$, so that $u_{n_{k}}$ converges weakly (for a subsequence) to $u^{*}$ in $L^{2}(\Omega), u_{n_{k}}^{+}$tends to $\tilde{u}$ in $H^{1}(\Omega)$ weakly and 
strongly in $L^{2}(\Omega)$ strongly, with $\tilde{u} \geq 0$. Let us define $u_{n_{k}}^{-}=u_{n_{k}}^{+}-u_{n_{k}}$, we immediatly get the weak convergence in $L^{2}(\Omega)$ of $u_{n_{k}}^{-}$towards $w=\tilde{u}-u^{*}$. Let $\chi$ be the characteristic function of $\{(x, y) \in \Omega, w(x, y)<0\}$, we have

$$
0 \leq \lim \int_{\Omega} \chi u_{n_{k}}^{-} d x d y=\int_{\Omega} \chi w d x d y \leq 0
$$

So that $w \geq 0 \quad$ a.e.

As

$$
\lim \int_{\Omega} u_{n_{k}}^{+} u_{n_{k}}^{-} d x d y=\int_{\Omega} \tilde{u} w d x d y=0, w \geq 0, \tilde{u} \geq 0
$$

We gain $w=u^{*^{-}}$and $\tilde{u}=u^{*^{+}}$.

Let us now prove that $u^{*}$ is a solution of the problem $\left(\mathcal{P}_{h^{*}}\right)$.

By substracting the right hand side of $\left(\mathcal{P}_{h_{n_{k}}}\right)$ and $\int_{\Omega}\left(h^{*}\right)^{3} \nabla u_{*}^{+} \nabla \phi-\int_{\Omega} h^{*}\left(1+\frac{1}{\beta} u_{*}\right) \frac{\partial \phi}{\partial x}$ for some $\phi \in V$, we obtain

$\left|\int_{\Omega} h_{n_{k}}^{3} \nabla u_{n_{k}}^{+} \cdot \nabla \phi-\int_{\Omega} h_{n_{k}}\left(1+\frac{1}{\beta} u_{n_{k}}\right) \frac{\partial \phi}{\partial x}-\int_{\Omega}\left(h^{*}\right)^{3} \nabla u_{*}^{+} \cdot \nabla \phi+\int_{\Omega} h^{*}\left(1+\frac{1}{\beta} u_{*}\right) \frac{\partial \phi}{\partial x}\right|$

$\leq\left|\int_{\Omega} h_{n_{k}}^{3}\left(\nabla u_{n_{k}}^{+}-\nabla u_{*}^{+}\right) \cdot \nabla \phi\right|+\left|\int_{\Omega} \nabla u_{*}^{+}\left(h_{n_{k}}^{3}-\left(h^{*}\right)^{3}\right) \cdot \nabla \phi\right|$

$+\left|\int_{\Omega} \frac{1}{\beta} h_{n_{k}}\left(u_{n_{k}}-u_{*}\right) \frac{\partial \phi}{\partial x}\right|+\left|\int_{\Omega}\left(1+\frac{1}{\beta} u_{*}\right)\left(h_{n_{k}}-h^{*}\right) \frac{\partial \phi}{\partial x}\right|-\int_{\Omega}\left(h_{n_{k}}-h^{*}\right) \frac{\partial \phi}{\partial x}$

As $h_{n_{k}} \in U_{a d}$, using (7) and the weak convergence of $\nabla u_{n_{k}}^{+}$, each term of the right hand side of the above inequality tends to zero, so we have :

$\int_{\Omega} h_{n_{k}}^{3} \nabla u_{n_{k}}^{+} \cdot \nabla \phi-\int_{\Omega} h_{n_{k}}\left(1+\frac{1}{\beta} u_{n_{k}}\right) \frac{\partial \phi}{\partial x} \rightarrow \int_{\Omega}\left(h^{*}\right)^{3} \nabla u_{*}^{+} \cdot \nabla \phi-\int_{\Omega} h^{*}\left(1+\frac{1}{\beta} u_{*}\right) \frac{\partial \phi}{\partial x}$

From the definition of the state equation $\left(\mathcal{P}_{h}\right)$, we immediately get that $u^{*}$ is a solution associated with the gap $h^{*}$. The next thing to prove is that

$$
\lim J\left(h_{n_{k}}, u_{n_{k}}\right)=J\left(h^{*}, u^{*}\right)
$$

As, $u^{+}\left(h_{n_{k}}\right) \rightarrow u_{*}^{+}$in $L^{2}(\Omega)$, an application of the Lebesgue's dominated convergence theorem implies:

$$
\left.\beta \log \left(1+\frac{1}{\beta} u_{n_{k}}^{+}\right)\right) \rightarrow \beta \log \left(1+\frac{1}{\beta} u_{*}^{+}\right) \text {in } L^{2}(\Omega) .
$$

So that $\left(h^{*}, u^{*}\right)$ is a solution of the problem $(\tilde{\mathcal{M}})$.

\section{Approached state equation}

It has been proved in [1] that a convenient way to obtain an existence theorem for $\left(\mathcal{P}_{h}\right)$ is to consider an approached problem $\left(\mathcal{P}_{h}\right)_{\eta \epsilon}$ depending on two small parameters $\epsilon>0, \eta>0$ so that the solution of $\left(\mathcal{P}_{h}\right)_{\eta \epsilon}$ tends to the one of $\left(\mathcal{P}_{h}\right)$ as $\epsilon$ and $\eta$ tend to zero. More precisely, $\epsilon$ and $\eta$ are related to two continuous approximations of the Heaviside graph namely $g_{\epsilon}(u)$ and $H_{\eta}(u)$ such that:

$$
g_{\epsilon}(u)= \begin{cases}1 & \text { if } u>0 \\ -\left(1-\frac{1}{\epsilon}\right) u+1 & \text { if }-\epsilon \leq u \leq 0 \\ \epsilon & \text { if } u<-\epsilon\end{cases}
$$




$$
H_{\eta}(u)=\left\{\begin{array}{l}
0 \text { if } u<0 \\
\frac{u}{\eta} \text { if } 0 \leq u \leq \eta \\
1 \text { if } u>\eta
\end{array}\right.
$$

and the following approached problem is defined by:

$$
\left(\mathcal{P}_{h}\right)_{\eta \epsilon}\left\{\begin{aligned}
& \text { Let } \theta_{0} \in L^{\infty}\left(\Gamma_{0}\right), 0 \leq \theta_{0} \leq 1, \text { find } u^{\eta \epsilon} \in V \text { such that } \\
& \int_{\Omega} h^{3} g_{\epsilon}\left(u_{\eta \epsilon}\right) \nabla u_{\eta \epsilon} \nabla \phi d x d y-\int_{\Omega} h H_{\eta}\left(1+\frac{1}{\beta} u_{\eta \epsilon}\right)\left(1+\frac{1}{\beta} u_{\eta \epsilon}\right) \frac{\partial \phi}{\partial x} d x d y \\
&=\int_{\Gamma_{0}} \Theta_{0} \phi d y \forall \phi \in V .
\end{aligned}\right.
$$

Let us define $G_{\epsilon}(t)=\int_{0}^{t} g_{\epsilon}(z) d z, G_{\epsilon}$

This is a continuous, increasing function so that a reciprocal function $G_{\epsilon}^{-1}$ exists. Using the classical Kirchoff transformation, (6) is rewritten as:

$$
\begin{aligned}
\int_{\Omega} h^{3} \nabla G_{\epsilon}\left(u_{\eta \epsilon}\right) \nabla \phi d x d y & -\int_{\Omega} h H_{\eta}\left(1+\frac{1}{\beta} u_{\eta \epsilon}\right)\left(1+\frac{1}{\beta} u_{\eta \epsilon}\right) \frac{\partial \phi}{\partial x} d x d y \\
& =\int_{\Gamma_{0}} \Theta_{0} \phi d y \forall \phi \in V .
\end{aligned}
$$

Let $v_{\eta \epsilon}=G_{\epsilon}\left(u_{\eta \epsilon}\right)$. A sequence of approached problems $\left(\mathcal{P}_{h}\right)_{\eta \epsilon}$ is defined as:

$$
\left(\mathcal{P}_{h}\right)_{\eta \epsilon}\left\{\begin{aligned}
& \text { Let } \Theta_{0} \in L^{\infty}\left(\Gamma_{0}\right), \Theta_{0} \geq 0, \text { find } v_{\eta \epsilon} \in V \text { such that: } \\
& \int_{\Omega} h^{3} \nabla v_{\eta \epsilon} \cdot \nabla \phi d x d y-\int_{\Omega} h T_{\eta \epsilon}\left(v_{\eta \epsilon}\right) \frac{\partial \phi}{\partial x} d x d y \\
&=\int_{\Gamma_{0}} \Theta_{0} \phi d y \quad \forall \phi \in V .
\end{aligned}\right.
$$

where

$$
T_{\eta \epsilon}(v):=H_{\eta}\left(1+\frac{1}{\beta} G_{\epsilon}^{-1}(v)\right)\left(1+\frac{1}{\beta} G_{\epsilon}^{-1}(v)\right)
$$

Remark 1 [1, Th. 2.14] : If (4) is satisfied, then $\left(\mathcal{P}_{h}\right)_{\eta \epsilon}$ admits a unique solution $v_{\eta \epsilon}$ such that

$$
\left\|v_{\eta \epsilon}\right\|_{H^{1}(\Omega)} \leq C,
$$

where $C$ is a constant which does not depend on $(\beta, \eta, \epsilon)$. There exists $u$ in $L^{2}(\Omega)$ such that $v_{\eta \epsilon}$ converges weakly to $u^{+}$in $H^{1}(\Omega)$ and $G_{\epsilon}^{-1}\left(v_{\eta \epsilon}\right)$ tends strongly to $u$ in $L^{2}(\Omega)$ so that

$$
\begin{array}{r}
u^{+}=\lim _{\eta \epsilon} v_{\eta \epsilon} \\
u^{-}=-\lim _{\eta \epsilon} G_{\epsilon}^{-1}\left(-v_{\eta \epsilon}^{-}\right)
\end{array}
$$

Moreover $u$ is a solution of $\left(\mathcal{P}_{h}\right)$.

Remark 2 The two parameters $\epsilon$ and $\eta$ have different meanings. The first one is a regularization parameter and the second one a penalization parameter. Moreover, the proof of convergence in [1] is such that $\eta$ tends to zero first and then $\epsilon$. So that one cannot choose $\eta=\epsilon$. Numerical results (5) showed that optimal choice are obtained by choosing $\eta \ll \epsilon$. 
Remark 3 As from $(1), v_{\eta \epsilon}$ tends to $u^{+}$in $H^{1}(\Omega)$, it is always possible to choose $\epsilon$ and $\eta$ so that the condition :

$$
1+\frac{1}{\beta} v_{\eta \epsilon}(h) \geq \nu>0 \text { a.e in } \Omega
$$

is fulfilled for a given $\nu, 0<\nu<1$. This condition will be used in the sequel.

Let $F_{\eta \epsilon}$ be the application defined from $V \times L^{\infty}(\Omega)$ to $H^{-1}(\Omega)$ by

$$
F_{\eta \epsilon}(v, h)=-\operatorname{div}\left(h^{3} \nabla v\right)+\frac{\partial\left(h T_{\eta \epsilon}(v)\right)}{\partial x},
$$

where $T_{\eta \epsilon}(v):=H_{\eta}\left(1+\frac{1}{\beta} G_{\epsilon}^{-1}(v)\right)\left(1+\frac{1}{\beta} G_{\epsilon}^{-1}(v)\right)$.

Proposition 1 : The mapping $(v, h) \mapsto F_{\eta \epsilon}(v, h)$ is continuously differentiable from $V \times L^{\infty}(\Omega)$ to $H^{-1}(\Omega)$

Proof Let $(w, g) \in V \times L^{\infty}(\Omega)$, with $(w, g) \neq 0$,

let us define

$$
\delta(w, g)=\frac{F_{\eta \epsilon}(v+w, h+g)-F_{\eta \epsilon}(v, h)-\left(L_{\eta \epsilon}(v, h), w, g\right)}{\|w\|_{V}+\|g\|_{L^{\infty}(\Omega)}},
$$

where $L_{\eta \epsilon}$ is the application defined from $V \times L^{\infty}(\Omega)$ to $\mathcal{L}\left(V \times L^{\infty}(\Omega), H^{-1}(\Omega)\right)$ by

$$
\begin{aligned}
\left(L_{\eta \epsilon}(v, h), w, g\right)= & -\operatorname{div}\left(3 h^{2} g \nabla v\right)+\frac{\partial\left(g T_{\eta \epsilon}(v)\right)}{\partial x} \\
& -\operatorname{div}\left(h^{3} \nabla w\right)+\frac{\partial\left(h \dot{T}_{\eta \epsilon}(v) w\right)}{\partial x},
\end{aligned}
$$

so that

$$
\begin{aligned}
& \left.\delta(w, g)=\frac{1}{\left(\|w\|_{V}+\|g\|_{L} \infty(\Omega)\right.}\right)\left(\frac{\partial\left(g\left(T_{\eta \epsilon}(v+w)-T_{\eta \epsilon}(v)\right)\right)}{\partial x}\right. \\
& -\operatorname{div}\left(\left((h+g)^{3}-h^{3}-3 h^{2} g\right) \nabla v\right)-\operatorname{div}\left(\left((h+g)^{3}-h^{3}\right) \nabla w\right) \\
& \left.+\frac{\partial\left(h\left(T_{\eta \epsilon}(v+w)-T_{\eta \epsilon}(v)-\dot{T}_{\eta \epsilon}(v) w\right)\right)}{\partial x}\right),
\end{aligned}
$$

and

$$
\begin{aligned}
\|\delta(w, g)\|_{H^{-1}(\Omega)} \leq & \frac{1}{\left(\|w\|_{V}+\|g\|_{L^{\infty}(\Omega)}\right)}\left(\left\|(h+g)^{3}-h^{3}-3 h^{2} g\right\|_{L^{\infty}(\Omega)}\|v\|_{V}\right. \\
& +\left\|(h+g)^{3}-h^{3}\right\|_{L^{\infty}(\Omega)}\|w\|_{V} \\
& +\|g\|_{L^{\infty}(\Omega)}\left\|T_{\eta \epsilon}(v+w)-T_{\eta \epsilon}(v)\right\|_{L^{2}(\Omega)} \\
& \left.+\|h\|_{L^{\infty}(\Omega)}\left\|T_{\eta \epsilon}(v+w)-T_{\eta \epsilon}(v)-\dot{T}_{\eta \epsilon}(v) w\right\|_{L^{2}(\Omega)}\right)
\end{aligned}
$$

as

$$
(h+g)^{3}-h^{3}-3 h^{2} g=g^{3}+3 h g^{2},
$$

then

$$
\begin{gathered}
\left\|(h+g)^{3}-h^{3}-3 h^{2} g\right\|_{L^{\infty}(\Omega)} \leq O\left(\|g\|_{L^{\infty}(\Omega)}\right)\|g\|_{L^{\infty}(\Omega)}, \\
\left\|(h+g)^{3}-h^{3}\right\|_{L^{\infty}(\Omega)} \leq O\left(\|g\|_{L^{\infty}(\Omega)}\right) .
\end{gathered}
$$


As $T_{\eta \epsilon}$ is Lipschitz and using the Poincare inequality, we get

$$
\left\|T_{\eta \epsilon}(v+w)-T_{\eta \epsilon}(v)\right\|_{L^{2}(\Omega)} \leq O\left(\|w\|_{V}\right),
$$

As $T_{\eta \epsilon}$ is continuously differentiable, there exists $\dot{T}_{\eta \epsilon}$ such that

$$
\left\|T_{\eta \epsilon}(v+w)-T_{\eta \epsilon}(v)-\dot{T}_{\eta \epsilon}(v) w\right\|_{L^{2}(\Omega)} \leq O\left(\|w\|_{V}\right)^{2},
$$

it follows that

$$
\|\delta(w, g)\|_{H^{-1}(\Omega)} \leq O\left(\|w\|_{V}+\|g\|_{L^{\infty}(\Omega)}\right),
$$

So, differentiability of $F_{\eta \epsilon}$ is gained with $\nabla F_{\eta \epsilon}(v, h)=L_{\eta \epsilon}(v, h)$.

In the same way, it can be proved that $\nabla F_{\eta \epsilon}$ is continuous from $V \times L^{\infty}(\Omega)$ to $\mathcal{L}\left(V \times L^{\infty}(\Omega), H^{-1}(\Omega)\right)$.

Proposition 2 : The mapping $h \mapsto v_{\eta \epsilon}(h)$ which defines the solution of $\left(\mathcal{P}_{h}\right)_{\eta \epsilon}$ is continuously differentiable from $\mathcal{U}_{\text {ad }}$ to $V$.

Proof The proof is based upon the implicit function theorem for the equation

$$
F_{\eta \epsilon}\left(v_{\eta \epsilon}(h), h\right)=0 .
$$

It is already known from Proposition 1 that $F_{\eta \epsilon}$ is continuously differentiable. The only thing to prove now is that

$$
\nabla_{1}\left(F_{\eta \epsilon}\left(v_{\eta \epsilon}(h), h\right)\right) \text { is an isomorphism from } V \text { to } H^{-1}(\Omega)
$$

where $\nabla_{1}$ denotes the derivative with respect to the first variable.

To do that, it is sufficient to prove that the linear problem

$$
\left\{\begin{array}{l}
z_{\eta \epsilon} \in H_{0}^{1}(\Omega) \\
-\operatorname{div}\left(h^{3} \nabla z_{\eta \epsilon}\right)+\frac{\partial\left(h \dot{T}_{\eta \epsilon}\left(v_{\eta \epsilon}\right) z_{\eta \epsilon}\right)}{\partial x}=f \text { in } H^{-1}(\Omega)
\end{array}\right.
$$

with $f \in H^{-1}(\Omega)$, admits a unique solution.

\section{Uniqueness:}

Let $z=z_{\eta \epsilon}^{1}-z_{\eta \epsilon}^{2}$ in which $z_{\eta \epsilon}^{1}$ and $z_{\eta \epsilon}^{2}$ are two solutions for (14) and $\gamma>0$ is a real given parameter. Multiplying the difference of the two equations satisfied by $z_{\eta \epsilon}^{1}$ and $z_{\eta \epsilon}^{2}$, by the test function $\frac{z^{+}}{\gamma+z^{+}}$which belongs to $H_{0}^{1}(\Omega)$, we get

$$
\int_{\Omega} h^{3} \nabla z \nabla\left(\frac{z^{+}}{\gamma+z^{+}}\right)=\int_{\Omega} h \dot{T}_{\eta \epsilon}\left(v_{\eta \epsilon}\right) z \frac{\partial}{\partial x}\left(\frac{z^{+}}{\gamma+z^{+}}\right),
$$

using the fact that

$$
\nabla\left(\frac{z^{+}}{z^{+}+\gamma}\right)=\gamma \frac{\nabla z^{+}}{\left(z^{+}+\gamma\right)^{2}}
$$

we get as $h$ belongs to $\mathcal{U}_{a d}$

$$
\int_{\Omega}\left|\frac{\nabla z^{+}}{\gamma+z^{+}}\right|^{2} \leq \frac{b}{a^{3}}\left\|\dot{T}_{\eta \epsilon}\left(v_{\eta \epsilon}\right)\right\|_{L^{\infty}(\Omega)} \int_{\Omega}\left|\frac{\frac{\partial}{\partial x} z^{+}}{\gamma+z^{+}}\right| .
$$


It follows, from the Poincaré inequality, that

$$
\int_{\Omega}\left(\log \left(1+\frac{z^{+}}{\gamma}\right)\right)^{2} \leq C
$$

where $C$ a constant that doesn't depend on $\gamma$.

Letting $\gamma$ tend to zero, we obtain $z \leq 0$ a.e in $\Omega$. Substituting $z$ for $-z$ we get $z \geq 0$ a.e in $\Omega$. The uniqueness is proved.

\section{Existence:}

Let $A_{\eta \epsilon}^{\varrho}$ be the application defined from $H_{0}^{1}(\Omega)$ to $H^{-1}(\Omega)$ by:

$$
A_{\eta \epsilon}^{\varrho}(v)=\varrho I v-\operatorname{div}\left(h^{3} \nabla v\right)+\frac{\partial\left(h \dot{T}_{\eta \epsilon}\left(v_{\eta \epsilon}\right) v\right)}{\partial x} .
$$

In which the operator $I: H_{0}^{1}(\Omega) \rightarrow H^{-1}(\Omega)$ is defined by

$$
\langle I v, w\rangle=\int_{\Omega} v w .
$$

We can choose $\varrho>0$ so that $A_{\eta \epsilon}^{\varrho}$ is coercive, and hence so that $\left(A_{\eta \epsilon}^{\varrho}\right)^{-1}$ exists and is continuous.

Let us remark that $z_{\eta \epsilon}$ is a solution of (14) if and only if

$$
z_{\eta \epsilon}-\varrho\left(A_{\eta \epsilon}^{\varrho}\right)^{-1} \circ I z_{\eta \epsilon}=\left(A_{\eta \epsilon}^{\varrho}\right)^{-1} f .
$$

As $H_{0}^{1}(\Omega)$ is compactly embedded in $L^{2}(\Omega)$, then $I$ is completely continuous. On the other hand $\varrho\left(A_{\eta \epsilon}^{\varrho}\right)^{-1}$ is continuous, so $\varrho\left(A_{\eta \epsilon}^{\varrho}\right)^{-1} \circ I$ is completely continuous. Applying the Fredholm's alternative [11] allows us to conclude.

\section{Approximate cost function and necessary optimality conditions}

To cope with the possible non uniqueness of the solution for the control problem, we introduce as in $[5,10]$ a modified cost function which forces the solution of the approximate problem to converge towards the solution $\left(h^{*}, u^{*}\right)$ deduced from theorem (2). Let us remark that usually, only one additional term is added. However possible non uniqueness of the state equation due to the weak regularity of the coefficient $h^{*}$ leads us to introduce another additional term containing $u^{*}$ in $J_{\eta \epsilon}(h)$.

The initial problem $(\mathcal{M})$ is approximated by:

$$
(\mathcal{M})_{\eta \epsilon}\left\{\begin{array}{l}
\text { Find } h_{\eta \epsilon}^{*} \in \mathcal{U}_{a d} \text { such that } \\
J_{\eta \epsilon}\left(h_{\eta \epsilon}^{*}\right)=\operatorname{Min}_{h \in \mathcal{U}_{a d}} J_{\eta \epsilon}(h)
\end{array}\right.
$$

where

$$
J_{\eta \epsilon}(h)=\frac{1}{2}\left\|\beta \log \left(1+\frac{1}{\beta} v_{\eta \epsilon}(h)\right)-P_{d}\right\|_{L^{2}(\Omega)}^{2}+\frac{1}{2}\left\|h-h^{*}\right\|_{L^{2}(\Omega)}^{2}+\frac{1}{2}\left\|v_{\eta \epsilon}(h)-u^{*}\right\|_{L^{2}(\Omega)}^{2}
$$

$v_{\eta \epsilon}(h)$ satifies $\left(\mathcal{P}_{h}\right)_{\eta \epsilon}$ and $\left(h^{*}, u^{*}\right)$ are given by theorem $(2)$. 
4.1 Necessary optimality conditions for the problem $(\mathcal{M})_{\eta \epsilon}$

Theorem 3 : There exists at least one optimal control $h_{\eta \epsilon}^{*} \in \mathcal{U}_{a d}$ for the problem $(\mathcal{M})_{\eta \epsilon}$ satisfying the following optimality system:

Let $\Theta_{0} \in L^{\infty}\left(\Gamma_{0}\right)$ and $\Theta_{0} \geq 0$,

$$
\left\{\begin{array}{l}
\text { find } v_{\eta \epsilon}^{*} \in V \text { such that for all } \phi \in V \\
\int_{\Omega}\left(h_{\eta \epsilon}^{*}\right)^{3} \nabla v_{\eta \epsilon}^{*} \nabla \phi d x d y-\int_{\Omega} h_{\eta \epsilon}^{*} T_{\eta \epsilon}\left(v_{\eta \epsilon}^{*}\right) \frac{\partial \phi}{\partial x} d x d y=\int_{\Gamma_{0}} \Theta_{0} \phi d y
\end{array}\right.
$$

and for all $h \in \mathcal{U}_{a d}$

$$
\int_{\Omega}\left(h-h_{\eta \epsilon}^{*}\right)\left(T_{\eta \epsilon}\left(v_{\eta \epsilon}^{*}\right) \frac{\partial q_{\eta \epsilon}}{\partial x}-3\left(h_{\eta \epsilon}^{*}\right)^{2} \nabla q_{\eta \epsilon} \nabla v_{\eta \epsilon}^{*}+h_{\eta \epsilon}^{*}-h^{*}\right) \geq 0,
$$

where $q_{\eta \epsilon}$ is a solution of the adjoint state equation

$$
\left\{\begin{array}{l}
q_{\eta \epsilon} \in H^{1}(\Omega) \text { such that }\left(q_{\eta \epsilon}\right) / \Gamma=0, \nabla q_{\eta \epsilon} \cdot \vec{n}_{/ \Gamma_{0}}=0 \text { and } \\
-\operatorname{div}\left(\left(h_{\eta \epsilon}^{*}\right)^{3} \nabla q_{\eta \epsilon}\right)-h_{\eta \epsilon}^{*} \dot{T}_{\eta \epsilon}\left(v_{\eta \epsilon}^{*}\right) \frac{\partial q_{\eta \epsilon}}{\partial x}=L_{\eta \epsilon}
\end{array}\right.
$$

where $L_{\eta \epsilon}=\frac{\beta \log \left(1+\frac{1}{\beta} v_{\eta \epsilon}^{*}\right)-P_{d}}{1+\frac{1}{\beta} v_{\eta \epsilon}^{*}}$.

Proof To prove that $(\mathcal{M})_{\eta \epsilon}$ admits at least one solution, the same arguments as for the proof of existence of a solution to $(\mathcal{M})$ are used.

To determine the adjoint system (17) it is sufficient to use the lagrangian:

$\mathcal{L}(h, v, q)=f(h, v)+\int_{\Omega}\left(h_{\eta \epsilon}^{*}\right)^{3} \nabla v \nabla q d x d y-\int_{\Omega} h_{\eta \epsilon}^{*} T_{\eta \epsilon}(v) \frac{\partial q}{\partial x} d x d y-\int_{\Gamma_{0}} \Theta_{0} q d y$,

where

$$
f(h, v)=\frac{1}{2}\left\|\beta \log \left(1+\frac{1}{\beta} v\right)-P_{d}\right\|_{L^{2}(\Omega)}^{2}+\frac{1}{2}\left\|h-h^{*}\right\|_{L^{2}(\Omega)}^{2}
$$

defined for $h \in \mathcal{U}_{a d}, v \in V$ satisfying the condition (11) and $q \in V$. The results of proposition (1) and (2) allow us to obtain (16) and (17).

4.2 Optimality conditions for the problem $(\mathcal{M})(1 \mathrm{D}$ case $)$

We will restrict ourselves to the one-dimensional case $\Omega=] 0,1[$ for which some estimates for the adjoint state can be established. The essential difficulty essentially comes from the term $h_{\eta \epsilon}^{*} \dot{T}_{\eta \epsilon}\left(v_{\eta \epsilon}^{*}\right) \frac{\partial q_{\eta \epsilon}}{\partial x}$ in equation (17).

In the following, we assume that

$$
P_{d} \in L^{\infty}(] 0,1[)
$$

Proposition 3 : Under the assumption (18) we have $\left\|q_{\eta \epsilon}\right\|_{L^{\infty}(] 0,1[)} \leq C$. 
Proof Let $Q=q_{\eta \epsilon}+k l(x)$, with $l(x)=\int_{0}^{x} \frac{z}{\left(h_{\eta \epsilon}^{*}\right)^{3}(z)} d z$, and $k$ a positive constant to be defined later.

we get

$$
\left(h_{\eta \epsilon}^{*}\right)^{3}(x) \frac{d l}{d x}=x \text { and } \frac{d}{d x}\left(\left(h_{\eta \epsilon}^{*}\right)^{3}(x) \frac{d l}{d x}\right)=1,
$$

then

$$
-\frac{d}{d x}\left(\left(h_{\eta \epsilon}^{*}\right)^{3}(x) \frac{d Q}{d x}\right)-h_{\eta \epsilon}^{*} \dot{T}_{\eta \epsilon}\left(v_{\eta \epsilon}^{*}\right) \frac{d Q}{d x}=Z_{\eta \epsilon}(k)
$$

with

$$
Z_{\eta \epsilon}(k)=L_{\eta \epsilon}^{*}-k\left(1+\frac{x \dot{T}_{\eta \epsilon}\left(v_{\eta \epsilon}^{*}\right)}{\left(h_{\eta \epsilon}^{*}\right)^{2}}\right)
$$

From definition (15), $v_{\eta \epsilon}^{*}$ satisfies the same kind of equation as $v_{\eta \epsilon}$ with $h_{\eta \epsilon}^{*}$ instead of $h$. As both $h_{\eta \epsilon}^{*}$ and $h$ verify (4), then $v_{\eta \epsilon}^{*}$ satisfies an estimate like (8).

Due to this estimate, using (11) and (18), we have

$$
\left\|L_{\eta \epsilon}\left(v_{\eta \epsilon}^{*}\right)\right\|_{L^{\infty}(] 0,1[)} \leq C
$$

Let us choose $k=\left.|| L_{\eta \epsilon}\left(v_{\eta \epsilon}^{*}\right)\right|_{L^{\infty}[0,1]}$, then $Z_{\eta \epsilon}(k) \leq 0$. By applying the maximum principle [24, Th 8.1], we get consequently $Q \leq \sup Q$, which implies that $\{0,1\}$

$$
q_{\eta \epsilon}(x)=Q(x)-k l(x) \leq \sup _{\{0,1\}} Q-k l(x) \leq C_{1}
$$

On the other hand the choice of $k=-\left\|L_{\eta \epsilon}\left(v_{\eta \epsilon}^{*}\right)\right\|_{L^{\infty}(] 0,1[)}$ implies that $Z_{\eta \epsilon}(k) \geq$ 0 . Using the same arguments we get $q_{\eta \epsilon}(x) \geq C_{2}$.

So $\left\|q_{\eta \epsilon}\right\|_{L^{\infty}(] 0,1[)} \leq C$.

Let us prove now the

Proposition 4 : If $h_{\eta \epsilon}^{*}$ is an optimal control of the problem $(\mathcal{M})_{\eta \epsilon}$, then $q_{\eta \epsilon}$ satisfies the following estimate

$$
\left\|q_{\eta \epsilon}\right\|_{H^{1}(] 0,1[)}+\left\|h_{\eta \epsilon}^{*} \dot{T}_{\eta \epsilon}\left(v_{\eta \epsilon}^{*}\right) \frac{\partial q_{\eta \epsilon}}{\partial x}\right\|_{L^{1}(] 0,1[)}+\left\|h_{\eta \epsilon}^{*} \dot{T}_{\eta \epsilon}\left(v_{\eta \epsilon}^{*}\right) \frac{\partial q_{\eta \epsilon}}{\partial x}\right\|_{H^{-1}(] 0,1[)} \leq C .
$$

Proof This is a direct consequence of 4 in which $a_{\epsilon}, u_{\epsilon}, g_{\epsilon}, L_{\epsilon}$ are respectively $h_{\eta \epsilon}^{*^{3}}, q_{\eta \epsilon}, h_{\eta \epsilon}^{*} \dot{T}_{\eta \epsilon}\left(v_{\eta \epsilon}^{*}\right) \frac{\partial q_{\eta \epsilon}}{\partial x}$ and $L_{\eta \epsilon}$. Assumption(40) is exactly (17) while assumptions $(41,42,43,44)$ are fulfilled by inequality (19), proposition 3 and the fact that $h_{\eta \epsilon}$ belongs to $\mathcal{U}_{a} d$.

In the one dimensional case, the following result will be proved: 
Lemma 1 There exists a constant $C$ which does not rely on $(\beta, \epsilon, \eta)$ such that the sequence $\left(v_{\eta \epsilon}\right)^{*}$ satisfies the estimation

$$
\left\|\frac{d v_{\eta \epsilon}^{*}}{d x}\right\|_{L^{\infty}(] 0,1[)} \leq C
$$

Proof Let $\varphi \in \mathcal{D}(] 0,1[)$. Then the function $\phi=\int_{1}^{x} \varphi(t) d t$ is a test function for (15) so we have

$$
\int_{0}^{1} h_{\eta \epsilon}^{*^{3}} \frac{d v_{\eta \epsilon}^{*}}{d x} \varphi d x=\int_{0}^{1} h_{\eta \epsilon}^{*} T_{\eta \epsilon}\left(v_{\eta \epsilon}^{*}\right) \varphi d x-\int_{0}^{1} \Theta_{0} \varphi d x
$$

so we deduce that

$$
\left.h_{\eta \epsilon}^{*^{3}} \frac{d v_{\eta \epsilon}^{*}}{d x}=h_{\eta \epsilon}^{*} T_{\eta \epsilon}\left(v_{\eta \epsilon}^{*}\right)-\Theta_{0} \quad \text { a.e } \quad \in\right] 0,1[
$$

Due to the one dimensional assumption the space $H^{1}(] 0,1[)$ is continuously imbedded in $L^{\infty}(] 0,1[)$ and from (8) the sequence $\left(v_{\eta \epsilon}^{*}\right)_{\eta \epsilon}$ is bounded in $L^{\infty}(] 0,1[)$. From the definition (7) of $T_{\eta \epsilon}$ we conclude that $\left(T_{\eta \epsilon}\left(v_{\eta \epsilon}^{*}\right)\right)_{\eta \epsilon}$ is bounded in $L^{\infty}(] 0,1[)$.

So the estimate (21) is obtained.

Proposition 5 For all $p \in\left[1, \infty\left[\right.\right.$ the sequence $\left(h_{\eta \epsilon}^{*}, v_{\eta \epsilon}^{*}\right)$ converges to $\left(h^{*}, u^{*^{+}}\right)$ defined by theorem 2 in $L^{p}(] 0,1[) \times H^{1}(] 0,1[)$ - weak) such that

$$
\int_{0}^{1} h^{*^{3}} \frac{d u^{*^{+}}}{d x} \frac{d \varphi}{d x} d x-\int_{0}^{1} h^{*}\left(1+\frac{1}{\beta} u^{*}\right) \frac{d \varphi}{d x} d x=\Theta_{0} \varphi(0) \forall \varphi \in V
$$

Proof As $\left(h_{\eta \epsilon}^{*}\right) \subset \mathcal{U}_{a d}$ then by (7) there exists $\tilde{h} \in \mathcal{U}_{a d}$ such that, after passing to a subsequence, $h_{\eta \epsilon}^{*}$ converges strongly to some $\tilde{h}$ in $L^{p}(] 0,1[)$ for all $p \in$ $[1, \infty[$.

As $h_{\eta \epsilon}^{*}$ is solution of the problem $(\mathcal{M})_{\eta \epsilon}$ we have

$$
J_{\eta \epsilon}\left(h_{\eta \epsilon}^{*}\right) \leq J_{\eta \epsilon}\left(h^{*}\right)
$$

and in the same way as in the end of the proof of theorem (2) we get

$$
\lim _{\eta, \epsilon \rightarrow 0} J_{\eta \epsilon}\left(h_{\eta \epsilon}^{*}\right)=\tilde{J}(\tilde{h}, \tilde{u})+\frac{1}{2}\left\|\tilde{h}-h^{*}\right\|_{L^{2}(\Omega)}^{2}+\frac{1}{2}\left\|\tilde{u}-u^{*}\right\|_{L^{2}(\Omega)}^{2}
$$

and from the definition of $\tilde{J}$

$$
\lim _{\eta, \epsilon \rightarrow 0} J_{\eta \epsilon}\left(h^{*}\right)=\tilde{J}\left(h^{*}, u^{*}\right)
$$

so passing to the limit in (25) we get

$$
\tilde{J}(\tilde{h}, \tilde{u})+\frac{1}{2}\left\|\tilde{h}-h^{*}\right\|_{L^{2}(\Omega)}^{2}+\frac{1}{2}\left\|\tilde{u}-u^{*}\right\|_{L^{2}(\Omega)}^{2} \leq \tilde{J}\left(h^{*}, u^{*}\right)
$$


Moreover, the definition of $\left(h^{*}, u^{*}\right)$ implies

$$
\tilde{J}\left(h^{*}, u^{*}\right) \leq \tilde{J}(\tilde{h}, \tilde{u})
$$

so that $\tilde{u}=u^{*}$ and $\tilde{h}=h^{*}$. As $u^{*}$ is unique, any subsequence $\left(v_{\eta \epsilon}\right)$ converges towards $u^{*}$.

By the same arguments as in the proof of $\left[1\right.$, Th. 2.14] the weak-limit $\tilde{u}^{+}$in $H^{1}(] 0,1[)$ of $\left(v_{\eta \epsilon}^{*}\right)$ is a solution of $\left(\mathcal{P}_{\tilde{h}}\right)$ and the solution $\tilde{u}$ of the limit problem is defined by $\tilde{u}^{+}=\lim _{\eta \epsilon}\left(v_{\eta \epsilon}\right)$ and $\tilde{u}^{-}=-\lim _{\eta \epsilon}\left(G_{\epsilon}^{-1}\left(-v_{\eta \epsilon}^{-}\right)\right)$.

In order to conclude, we need to prove the strong $H^{1}$ convergence of $v_{\eta \epsilon}^{*}$ towards $u^{*^{+}}$. To do that, we will adopt a two step procedure, letting first $\eta$ tend to zero and then $\epsilon$. The strong convergence will be proved for each of the two steps, so inducing the result.

Proposition 6 As $\eta$ tends to zero, there exists $v_{\epsilon}^{*}$ and $h_{\epsilon}^{*}$ strong limits in $H^{1}(] 0,1[)$ and in $L^{p}(] 0,1[)$ for all $1 \leq p \leq \infty$ of $v_{\eta \epsilon}^{*}$ and $h_{\eta \epsilon}^{*}$ such that

$$
\int_{\Omega} h_{\epsilon}^{*^{3}} \nabla v_{\epsilon}^{*} \cdot \nabla \phi d x-\int_{\Omega} h_{\epsilon}^{*}\left(1+\frac{1}{\beta} G_{\epsilon}^{-1}\left(v_{\epsilon}^{*}\right)\right)^{+} \frac{\partial \phi}{\partial x} d x=\Theta_{0} \phi(0) \quad \forall \phi \in V .
$$

Proof From appendix 3 and estimate (21), there exists a weak limit $v_{\epsilon}^{*}$ in $H^{1}$ and a strong limit in $L^{p}(] 0,1[)$ of $v_{\eta \epsilon}^{*}$ and $h_{\eta \epsilon}^{*}$ as $\eta$ tends to zero. Moreover, let us remark that for any sequence $w_{\eta}$ which strongly converges in $L^{2}(] 0,1[)$ to $w$, we get

$$
H_{\eta}\left(w_{\eta}\right) \cdot w_{\eta} \longrightarrow w^{+} \text {strongly in } L^{2}(] 0,1[)
$$

Due to the definition (7) of $T_{\eta \epsilon}$ and as the convergence of $v_{\eta \epsilon}^{*}$ implies the $L^{2}$-strong convergence of $G_{\epsilon}^{-1}\left(v_{\eta \epsilon}^{*}\right)$, then using (27) and passing to the limit in $\eta$ in equation (15), we get exactly (26).

To prove the strong convergence of $v_{\eta \epsilon}^{*}$ to $v_{\epsilon}^{*}$, we write using (15):

$$
\begin{aligned}
\int_{\Omega} h_{\epsilon}^{*^{3}}\left(\frac{d\left(v_{\eta \epsilon}^{*}-v_{\epsilon}^{*}\right)}{d x}\right)^{2} d x & =\int_{\Omega}\left(h_{\epsilon}^{*^{3}}-h_{\eta \epsilon}^{*^{3}}\right)\left(\frac{d\left(v_{\eta \epsilon}^{*}-v_{\epsilon}^{*}\right)}{d x}\right)^{2} d x+\int_{\Omega}\left(h_{\epsilon}^{*}-h_{\eta \epsilon}^{*}\right) T_{\eta \epsilon}\left(v_{\eta \epsilon}\right) \frac{d\left(v_{\eta \epsilon}^{*}-v_{\epsilon}^{*}\right)}{d x} d x \\
& -\int_{\Omega} h_{\epsilon}^{*} T_{\eta \epsilon}\left(v_{\eta \epsilon}\right) \frac{d\left(v_{\eta \epsilon}^{*}-v_{\epsilon}^{*}\right)}{d x} d x
\end{aligned}
$$

The result follows immediately from (21), the strong convergence of $h_{\eta \epsilon}^{*}$ and the fact that $T_{\eta \epsilon}\left(v_{\eta \epsilon}\right)$ is bounded in $L^{\infty}(] 0,1[)$.

For the sequence $\left(v_{\epsilon}\right)$ the following lemma can also be proved :

Lemma 2 The sequence $\left(v_{\epsilon}\right)$ verifies

$$
\lim _{\epsilon \rightarrow 0} \int_{\Omega}\left|\nabla v_{\epsilon}^{*^{-}}\right|^{2} d x=0
$$


Proof Choosing $v_{\epsilon}^{*^{-}}$as a test function in (26) we obtain

$$
-\int_{\Omega} h_{\epsilon}^{3}\left|\nabla v_{\epsilon}^{*^{-}}\right|^{2}=\int_{\Omega} h_{\epsilon}\left(1+\frac{1}{\beta} G_{\epsilon}^{-1}\left(v_{\epsilon}^{*}\right)\right)^{+} \frac{\partial v_{\epsilon}^{*^{-}}}{\partial x} d x+\Theta_{0} v_{\epsilon}^{*^{-}}(0)
$$

From the definition of $G_{\epsilon}$ we get

$$
0 \leq\left(1+\frac{1}{\beta} G_{\epsilon}^{-1}\left(v_{\epsilon}^{*}\right)\right) \leq 1
$$

and

$$
G_{\epsilon}^{-1}\left(v_{\epsilon}^{*}\right)=G_{\epsilon}^{-1}\left(v_{\epsilon}^{*^{+}}\right)+G_{\epsilon}^{-1}\left(-v_{\epsilon}^{*^{-}}\right)=v_{\epsilon}^{*^{+}}+G_{\epsilon}^{-1}\left(-v_{\epsilon}^{*^{-}}\right)
$$

so we can rewrite $(29)$ as

$$
-\int_{\Omega} h_{\epsilon}^{*^{3}}\left|\frac{d v_{\epsilon}^{*^{-}}}{d x}\right|^{2}=\int_{\Omega} h_{\epsilon}^{*} \frac{d v_{\epsilon}^{*^{-}}}{d x} d x+\int_{\Omega} h_{\epsilon}^{*} \frac{1}{\beta} G_{\epsilon}^{-1}\left(-v_{\epsilon}^{*^{-}}\right) \frac{d v_{\epsilon}^{*^{-}}}{d x} d x+\Theta_{0} v_{\epsilon}^{*^{-}}
$$

Due to proposition 5 the sequence $\left(v_{\epsilon}^{*}\right)$ converges, up to a subsequence, to $u^{*^{+}}$ in $H^{1}(\Omega)$ weak and strongly in $L^{2}(\Omega)$ where $u^{*}$ is a solution of the problem $\left(\mathcal{P}_{h^{*}}\right)$. The first term of the right hand side in $(32)$ tends to 0 and from the compact inclusion of $H^{1}(\Omega)$ in $C^{0}(\bar{\Omega})$ the third term also tends to 0 . To study the limit of the term $\int_{\Omega} h_{\epsilon} \frac{1}{\beta} G_{\epsilon}^{-1}\left(-v_{\epsilon}^{-}\right) \frac{d v_{\epsilon}^{*}}{d x} d x$, the function $B_{\epsilon}(x)=$ $\int_{0}^{x} G_{\epsilon}^{-1}(t) d t$ is introduced. We have:

$$
\int_{\Omega} h_{\epsilon} \frac{1}{\beta} G_{\epsilon}^{-1}\left(-v_{\epsilon}^{*^{-}}\right) \frac{d v_{\epsilon}^{*^{-}}}{d x} d x=-\int_{\Omega} h_{\epsilon}^{*} \frac{d B_{\epsilon}\left(-v_{\epsilon}^{*^{-}}\right)}{d x} d x
$$

As $h_{\epsilon}^{*} \in B V(\Omega)$ and $B_{\epsilon}$ is continuous we can now apply the formula for integration by parts [28, page 297-298] to obtain

$\int_{\Omega} h_{\epsilon}^{*} \frac{d B_{\epsilon}\left(-v_{\epsilon}^{*^{-}}\right)}{d x} d x=-\int_{\Omega} B_{\epsilon}\left(-v_{\epsilon}^{*^{-}}\right) D h_{\epsilon}+h_{\epsilon}^{*}(1) B_{\epsilon}\left(-v_{\epsilon}^{*^{-}}\right)(1)-h_{\epsilon}^{*}(0) B_{\epsilon}\left(-v_{\epsilon}^{*^{-}}\right)(0)$

in the sens of Riemann-Stieltjes and as $B_{\epsilon}(0)=0$, we have

$$
\int_{\Omega} h_{\epsilon}^{*} \frac{d B_{\epsilon}\left(-v_{\epsilon}^{*^{-}}\right)}{d x} d x=-\int_{\Omega} B_{\epsilon}\left(-v_{\epsilon}^{*^{-}}\right) D h_{\epsilon}^{*}-h_{\epsilon}^{*}(0) B_{\epsilon}\left(-v_{\epsilon}^{*^{-}}(0)\right)
$$

from $(30)$ we have

$$
\left|B_{\epsilon}\left(-v_{\epsilon}^{*^{-}}\right)\right| \leq \beta\left|-v_{\epsilon}^{*^{-}}\right| \text {and }\left|B_{\epsilon}\left(-v_{\epsilon}^{*^{-}}(0)\right)\right| \leq \beta\left|v_{\epsilon}^{*^{-}}(0)\right|
$$

using the definition of $\mathcal{U}_{a d}$ and the compact embedding of $H^{1}(\Omega)$ into $C^{0}(\bar{\Omega})$ :

$$
\left|\int_{\Omega} h_{\epsilon}^{*} \frac{d B_{\epsilon}\left(-v_{\epsilon}^{*^{-}}\right)}{d x} d x\right| \leq \beta\left(\left|D h_{\epsilon}^{*}\right|+b\right)\left\|v_{\epsilon}^{-}\right\|_{C^{0}[0,1]}
$$

SO

$$
\lim _{\epsilon \rightarrow 0} \int_{\Omega} h_{\epsilon}^{*} \frac{d B_{\epsilon}\left(-v_{\epsilon}^{*^{-}}\right)}{d x} d x=0
$$


Theorem 4 The sequence $\left(v_{\epsilon}^{*}\right)$ and in turn $\left(v_{\eta \epsilon}^{*}\right)$ converges strongly to $u^{*}$ in $H^{1}(\Omega)$.

Proof The $H^{1}$ weak convergence of $\left(v_{\epsilon}^{*}\right)$ towards $u^{*^{+}}$comes from proposition 5 and estimate 21. By making the difference between equations (26) and (24) and choosing $v_{\epsilon}^{*}-u^{*^{+}}$as a test function we get

$$
\begin{aligned}
\int_{\Omega} h^{*^{3}}\left(\frac{d v_{\epsilon}^{*}}{d x}-\frac{d u^{*^{+}}}{d x}\right)^{2} d x= & \int_{\Omega}\left(h^{*^{3}}-h_{\epsilon}^{*^{3}}\right)\left(\frac{d v_{\epsilon}^{*}}{d x}-\frac{d u^{*^{+}}}{d x}\right) \frac{d v_{\epsilon}^{*}}{d x} d x \\
+ & \int_{\Omega}\left(h^{*}-h_{\epsilon}^{*}\right)\left(1+\frac{1}{\beta} G_{\epsilon}^{-1}\left(v_{\epsilon}^{*}\right)\right)\left(\frac{d v_{\epsilon}^{*}}{d x}-\frac{d u^{*}}{d x}\right) d x \\
& +\frac{1}{\beta} \int_{\Omega} h^{*}\left(G_{\epsilon}^{-1}\left(v_{\epsilon}^{*}\right)-u^{*^{+}}\right)\left(\frac{d v_{\epsilon}^{*}}{d x}-\frac{d u^{*}}{d x}\right) d x
\end{aligned}
$$

Due to the fact that $h^{*}$ and $h_{\epsilon}^{*}$ belong to $\mathcal{U}_{a d}$, the sequence $\left(h_{\epsilon}^{*}\right)$ converges to $h^{*}$ in $L^{p}(\Omega)$ for all $1 \leq p<\infty$. From estimate $(21)$ the first and second terms of the right hand side of the equality go to zero as $\epsilon$ tends to zero. For the last term, it can be rewritten as:

$$
\begin{aligned}
I=\frac{1}{\beta} \int_{0}^{1} h^{*}\left(G_{\epsilon}^{-1}\left(v_{\epsilon}^{*}\right)-u^{*^{+}}\right)\left(\frac{d v_{\epsilon}^{*^{+}}}{d x}-\right. & \left.\frac{d u^{*^{+}}}{d x}\right) d x-\frac{1}{\beta} \int_{0}^{1} h^{*}\left(G_{\epsilon}^{-1}\left(v_{\epsilon}^{*}\right)-u^{*^{+}}\right) \frac{d v_{\epsilon}^{*^{-}}}{d x} d x \\
& =I_{1}+I_{2}
\end{aligned}
$$

by lemma $2, I_{2}$ tends to zero using (31).

Let us rewrite $I_{1}$ :

$I_{1}=\frac{1}{\beta} \int_{\Omega} h^{*}\left(v_{\epsilon}^{*^{+}}-u^{*^{+}}\right)\left(\frac{d v_{\epsilon}^{*^{+}}}{d x}-\frac{d u^{*^{+}}}{d x}\right) d x-\frac{1}{\beta} \int_{\Omega} h^{*}\left(G_{\epsilon}^{-1}\left(-v_{\epsilon}^{*^{-}}\right)-u^{*^{-}}\right)\left(\frac{d v_{\epsilon}^{*^{+}}}{d x}-\frac{d u^{*^{+}}}{d x}\right) d x$

since $\left(v_{\epsilon}^{*^{+}}\right)$converges strongly to $u^{*^{+}}$in $L^{2}(\Omega)$ and weakly in $H^{1}(] 0,1[)$, the first term in the above equality tends to 0 and the second can be rewritten as

$\frac{1}{\beta} \int_{\Omega} h^{*}\left(G_{\epsilon}^{-1}\left(-v_{\epsilon}^{*^{-}}\right)-u^{*^{-}}\right)\left(\frac{d v_{\epsilon}^{*^{+}}}{d x}-\frac{d u^{*^{+}}}{d x}\right) d x=\frac{1}{\beta} \int_{0}^{1} h^{*}\left(G_{\epsilon}^{-1}\left(-v_{\epsilon}^{*^{-}}\right) \frac{d u^{*^{+}}}{d x}\right) d x+\frac{1}{\beta} \int_{\Omega} h^{*} u^{*^{-}} \frac{d v_{\epsilon}^{*^{+}}}{d x} d x$

Using the same arguments as in remark 1, (10) and proposition 5 we then deduce that this term tends to

$$
\frac{1}{\beta} \int_{0}^{1} h^{*} u^{*^{-}} \frac{d u^{*^{+}}}{d x} d x+\frac{1}{\beta} \int_{\Omega} h^{*} u^{*^{-}} \frac{d u^{*^{+}}}{d x} d x
$$

which is zero.

The strong $H^{1}$ convergence of $\left(v_{\epsilon}^{*}\right)$ towards $u^{*^{+}}$is proved and in turn that of $v_{\eta \epsilon}^{*}$ by using the triangle inequality. 
Theorem 5 : Let $\left(h^{*}, u^{*}\right)$ be an optimal control of the problem $(\tilde{\mathcal{M}})$. Then there exist $(q, \lambda)$ in $H^{1}(] 0,1[) \times M(] 0,1[)$ such that

$$
\int_{] 0,1[} h^{*^{3}} \frac{d u^{*^{+}}}{d x} \frac{d \phi}{d x} d x-\int_{] 0,1[} h^{*}\left(1+\frac{1}{\beta} u^{*}\right) \frac{d \phi}{d x} d x=\Theta_{0} \phi(0) \forall \phi \in V,
$$

$\forall h \in \mathcal{U}_{a d}$

$$
\begin{gathered}
\int_{] 0,1[}\left(h-h^{*}\right)\left(\left(1+\frac{1}{\beta} u^{*}\right) \frac{d q}{d x}-3 h^{*^{2}} \frac{d q}{d x} \frac{d u^{*^{+}}}{d x}\right) d x \geq 0, \\
\left\{\begin{array}{l}
q \in V, \\
\int_{] 0,1[} h^{*^{3}} \frac{d q}{d x} \frac{d \phi}{d x} d x-\int_{] 0,1[} \lambda \phi d x=\int_{] 0,1[} \frac{\beta \log \left(1+\frac{1}{\beta} u^{*^{+}}\right)-P_{d}}{1+\frac{1}{\beta} u^{*^{+}}} \phi d x, \forall \phi \in V,
\end{array}\right.
\end{gathered}
$$

with $M(] 0,1[)=\left(C_{0}(] 0,1[)\right)^{*}$ the space of bounded measures in $] 0,1[$.

Proof According to the estimate (20) there exist two multipliers $q$ and $\lambda$ in $H^{1}(] 0,1[)$ and $M(] 0,1[)$ respectively, such that $q_{\eta \epsilon}$ converges weakly to $q$ in $H^{1}(] 0,1[), \lambda_{\eta \epsilon}$ converges to $\lambda$ in $H^{-1}(] 0,1[)$-weakly and $M(] 0,1[)-{ }^{*}$-weakly.

Due to Remark 1 , there exists $u$ in $L^{2}(] 0,1[)$ such that $v_{\eta \epsilon}^{*}$ converges weakly to $u^{*^{+}}$in $H^{1}(] 0,1[)$ and $G_{\epsilon}^{-1}\left(v_{\eta \epsilon}^{*}\right)$ tends to $u^{*}$ in $L^{2}(] 0,1[)$.

Passing to the limit with respect to $\eta$ and $\epsilon$ in (15)-(17), we obtain (35)-(37).

\section{Numerical realization}

The theory in the previous sections has been mainly developped in the context of the hydrodynamic lubrication(The Reynolds state is the governing equation for thin film flow). The one-dimensional assuption for which optimality conditions have been obtained in section 4 can be associated with a bearing (see figure 1) whose diameter is small with respect of its width: the so called infinitely long bearing [23]. In that case the flow is roughly independent of the variable $y$ (in reality there is a small boundary layer on both ends of the bearing) and the one dimensional Reynolds equation is used to get the pressure inside the device. As it will be shown in the following figures, the pressure reaches its maximal value around the middle of the device where the gap is minimal. This value can be so high that the surface of the device can be locally deformed [16] and a flat part appears: the original shape is truncated. In most of the applications, this phenomenon is amplified by the variation of the viscosity with the pressure (piezoviscosity). This phenomenon is not taken into account in the present work as the state Reynolds equation that is dealt with is highly non linear. Thus the related inverse problem is not included in the present study. Nevertheless, we will retain as a numerical illustration of the present theory the identification of a locally deformed surface $h$ by the knowledge of the pressure field $P_{d}$. 


\subsection{Initialization:}

Assuming the gap $h$ is known, the pressure $P_{d}=\beta \log \left(1+\frac{u_{d}^{+}}{\beta}\right)$ will be introduced by the following procedure to prevent the so called "inverse crime problem":

- It can be proved as in [6] that for suitable data the solution of (3) for a journal bearing geometry is a continuous function. Moreover the non cavitated area is made of two (unknown) parts, namely $[0, A]$ and $[B, 1]$. The first one is located in the convergent part of the bearing, the second one in the divergent part. In addition $\frac{d P}{d x}=0$ on $x=B$ so that $\rho=1$ at this point. The mass flow conservation in $[B, 1]$ gives:

$$
h(B) \rho(B)=h(B)=\Theta_{0}
$$

This allows us to compute the location of $B$ as soon as $\min (h)_{[0,1]} \leq \Theta_{0} \leq$ $\max (h)_{[0,1]}$.

- A backward ordinary differential equation iterative procedure is carried out from $x=B$ to solve the Reynolds equation

$$
-h^{3} \frac{d u^{+}}{d x}+\left(1+\frac{1}{\beta} u\right) h=\Theta_{0}
$$

This procedure is stopped at a point $A$ where the pressure becomes non positive. The overall solution is defined by: $u_{d}(x)=u(x)$ for $A \leq x \leq B$ and $u(x)=0$ elsewhere.

5.2 Choice of the penalization parameters and the datas

To identify the deformed thickness $h_{d}(x)$ from the desired pressure $p_{d}$ computed by the above procedure we use the optimality system (15)-(17). Equation (15) is solved using the $P 1$ finite elements and fixed-point procedure. We then solve the corresponding adjoint problem (17) and we evaluate the gradient of $J_{\eta \epsilon}(16)$. To minimize $J_{\eta \epsilon}$ on $\mathcal{U}_{a d}$ we take a regularized descent direction $D_{\eta \epsilon}$ obtained as a solution of the elliptic problem

$$
\left\{\begin{array}{l}
-\Delta D_{\eta \epsilon}+r D_{\eta \epsilon}=-\nabla\left(J_{\eta \epsilon}\right) \quad \text { in } \Omega \\
\frac{\partial D_{\eta \epsilon}}{\partial n}=0 \quad \text { on } \partial \Omega
\end{array}\right.
$$

where $r$ is a parameter which has to be adjusted numerically to accelerate the convergence of the projected BFGS with Armijo rule [26].

For tests below the exact deformed thikness is

$$
h_{d}(x)= \begin{cases}1+0.5 \cos (2 \pi x) & \text { if } x \in([0,1]-[0.4,0.6]) \\ 0.6 & \text { if } x \in([0,1]-[0.4,0.6])\end{cases}
$$

The choice of the values of the parameters deserves further discussions. The value chosen for $\beta$ is a usual value for the bulk modulus of a lubricant [32]. The 
values of $\eta$ and $\epsilon$ have been chosen to obtain the best stability with respect to the number of grid's points.

The initial thickness is $h(x)=1+0,5 \cos (2 \pi x), \beta=1.10^{9}, \eta=10^{-5}$ and $\epsilon=2.10^{-3}$

The value $\Theta_{0}=0.675$ for the input mass flow is chosen so that two cavitation

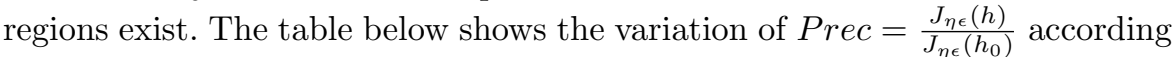
to the NIT number of iterations and for different values of the number $m$ of of grid's points.

\begin{tabular}{|c||c||c||c||c|}
\hline & $m=101$ & $m=201$ & $m=301$ & $m=401$ \\
\hline NIT & Prec & Prec & Prec & Prec \\
\hline 1 & $1.0000 E+00$ & $1.0000 E+00$ & $1.0000 E+00$ & $1.0000 E+00$ \\
\hline 5 & $1.52571 e-03$ & $2.43188 e-03$ & $2.12803 e-03$ & $2.32768 e-03$ \\
\hline 10 & $1.34161 e-03$ & $1.23072 e-03$ & $1.06685 e-03$ & $1.15125 e-03$ \\
\hline 20 & $1.33785 e-03$ & $1.38974 e-04$ & $4.75835 e-04$ & $4.50564 e-04$ \\
\hline 40 & $1.33785 e-03$ & $1.84877 e-05$ & $1.23317 e-04$ & $1.70753 e-04$ \\
\hline
\end{tabular}

Table 1
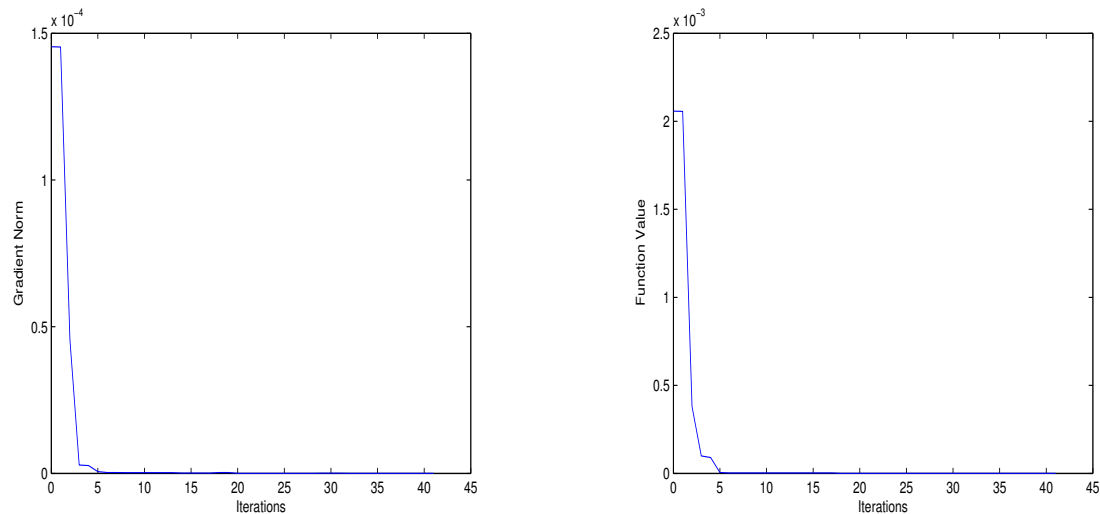

Fig. 2

Fig. 2 shows the good convergence of the projected-BFGS algorithm combined with our choice of the regularized descent direction $D_{\eta \epsilon}$ for $m=201$.

Fig. 3 and Fig. 4 depict a comparaison between the computed pressure and thickness profiles and the exact ones for $m=401$ after various number of iterations. 


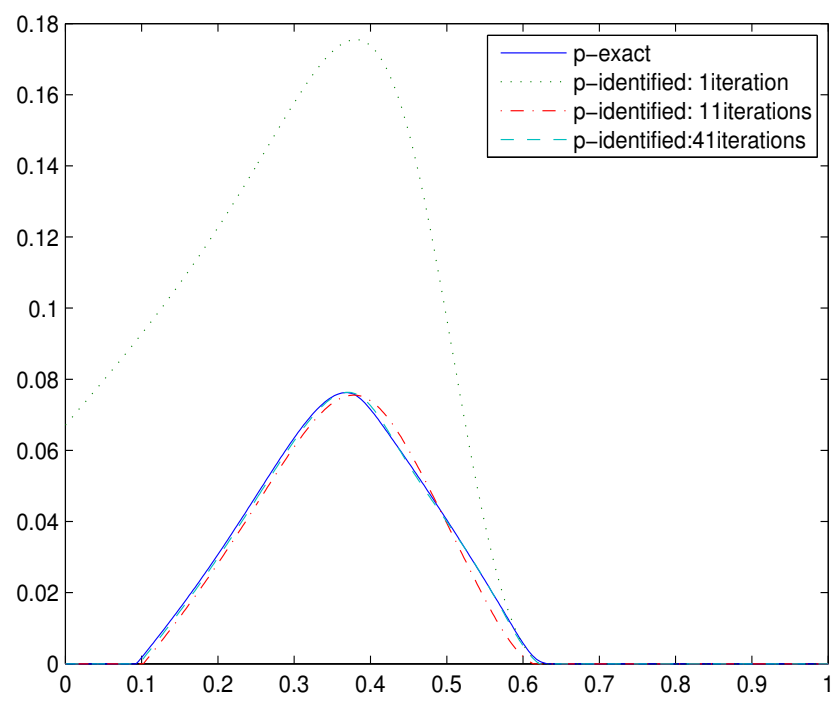

Fig. 3

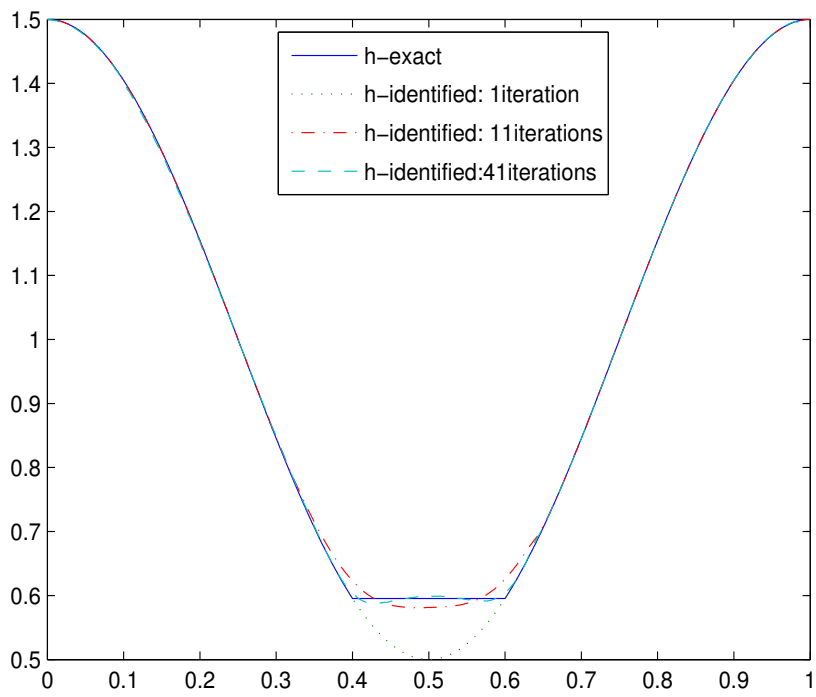

Fig. 4

\section{Conclusion}

The present work shows how is it possible to get some identification process for thin film flow taking a correct mass preserving condition into account. It 
opens the way to optimization problems in the same mechanical area, as finding the best shape (the function $h$ ) to minimize friction or oil consumption. An interesting feature is that recent physical studies of the gaseous cavitation in lubrication tends to give a physical meaning to some mathematical problems close to the approximate one (6)[7] used in the present paper. This induces an additional reason to introduce more efficient numerical methods for solving both direct and inverse problems.

Acknowledgements This work was partially supported by the French-Morocco cooperation CNRS-CNRST ( SPM02/12 ) and the CMIMF action intégrée (MA/04/94).

\section{References}

1. Ait Hadi, K., Bayada, G., El Alaoui Talibi, M.: Existence and uniqueness for a non coercive lubrication problem. J. Math. Anal. Appl. 327(1), 585-610 (2007)

2. Alvarez, S.J., Carrillo, J.: A free-boundary problem in theory of lubrication. Commun. Partial Differential Equations 19, 11-12, 1743-1761 (1994)

3. Auloge, J.Y., Bourguin, P., Gay, B.: The optimum design of onedimensional bearings with non-newtonian lubricants. Journal of Lubrication Technology 105, 391-395 (1983)

4. Ausas, R., Ragot, P., Leiva, J., Jai, M., Bayada, G., Buscaglia, G.: The impact of the cavitation model in the analysis of micro textured lubricated journal bearings. ASME Journal of Tribology 129, 1219-1234 (2007)

5. Barbu, V.: Necessary conditions for non convex distributed control problems governed by variational inequalities. J. Math. Anal. Appl. 80, 566-598 (1981)

6. Bayada, G., Chambat, M.: Analysis of a free boundary problem in partial lubrication. Quarterly of applied math XL(4), 372 (1983)

7. Bayada, G., Chupin, L.: Thin film compressible model and JakobssonFloberg-Olsson/Elrod-Adams conservative cavitation model. Submitted to ASME Journal of Tribology (2012)

8. Bayada, G., El Alaoui Talibi, M.: An application of the control by coefficients in a variational inequality for hydrodynamic lubrication. Nonlinear Analysis 1, 315-328 (2000)

9. Bayada, G., Meurisse, M.H.: The impact of the cavitation model on the theoritical performance of heteregenous slip/no enginered contact. Proc. Inst. Mech. Eng., part J, Journal. Eng. Tribology 223, 3, 371-381 (2009)

10. Bermudez, A., Saguez, C.: Optimal control of variational inequalities. Control and Cybernetics 14(1-3), 9-30 (1985)

11. Brezis, H.: Analyse fonctionnelle Théorie et applications, Masson edn. (1983) 
12. C.H. Cheng, M.H. Chang: Shape design for surface of a slider by inverse method. J. Tribol. 126(3), 519-526 (2004)

13. Chipot, M.: Variational Inequalities and Flow in Porous Media, SpringerVerlag, New York Inc edn. (1984)

14. Chupin, L., Sart, R.: Compressible flows: New existence results and justification of the reynolds asymptotic in thin film. Asymptotic Analysis 76 (3-4), 193-291 (2012)

15. Ciuperca, I., El Alaoui Talibi, M., Jai, M.: On the optimal control of coefficients in elliptic problems. application to the optimization of the head slider subject to bounds. ESAIM Control Optim. Calc. Var. 11(1), 102-121 (2005)

16. Dowson, D., Higginson, G.R.: Elasto hydrodynamic lubrication, International series in material science and technology, vol. 23, Pergamon press edn., p. 80 (1977)

17. El Alaoui Talibi, M., Kacimi, E.: A constraints relaxation technique for solving an identification of coefficients problem issued from hydrodynamic lubrication. J. Inverse Ill-Posed Probl. 10(3), 295-318 (2002)

18. El Alaoui Talibi, M., Ouazar, D., Tber, M.: Identification of the hydraulic conductivities in a saltwater intrusion problem. J. Inv. Ill-Posed Problems 15, 1-20 (2007)

19. El Kacimi, A.: Contrôle optimal de problèmes à frontière libre et quelques applications en lubrification elastohydrodynamique. Ph.D. thesis, Faculty of sciences Semlalia, Cadi Ayyad University, Marrakesh, Morocco (2001)

20. Elrod, H.G., Adams, M.L.: A computer program for cavitation in related phenomena in lubrication. In: Proceedings, Mech. Eng. Publ. td., pp. 37-42 (1975)

21. Elsharkawy, A.A., Guedouar, L.H.: Direct and inverse solutions for elastohydrodynamic lubrication of finite porous journal bearings. Transactions of the ASME J. Tribol. 123(2), 276-282 (2001)

22. Evans, L.C., Gariepy, R.F.: Measure theory and fine properties of functions, CRC Press, Florida edn. Studies in Advanced Mathematics (1992)

23. Frene, J., Nicolas, D., Degueurce, B., Berthe, D., Godet, M.: Lubrification hydrodynamique, Paliers et Butées, Eyrolles Paris edn. (1990)

24. Gilbarg, D., Trudinger, N.S.: Elliptic partial differential equations of second order, 2nd ed., springer-verlag, berlin edn. (1983)

25. Jakobson, B., Floberg, L.: The finite journal bearing considering vaporization. Trans. Chalmers Univ. Tech. 190, 1-66 (1957)

26. Kelley, C.: Iterative Methods for Optimization, Frontiers in Applied Mathematics, SIAM edn. (1999)

27. Kinderleher, D., Stampacchia, G.: An introduction to variational inequalities and applications, academic press edn. (1980)

28. Lang, S.: Real and Functional Analysis, Springer edn. (1993)

29. Maday, C.J.: The maximum principle approach to the optimal 1.dimensional journal bearing. ASME J. Lub. Tech 92, 482-489 (1970) 
30. Meurisse, M.H.: Solution of the inverse problem in hydrodynamic lubrication. pp. 104-107. Inst. Mech. Eng London (1983)

31. Rayleigh, L.: Notes on the theory of lubrication. Philos Mag 35, 1-12 (1918)

32. Sahlin, F., Almquist, A., Larsson, R., Glavatskih, S.: A cavitation algorithm for arbitrary lubricant compressibility. Tribology International 40, 1294-1300 (2007) 
A Some properties of BV-Spaces[22]

Lemma 3 :

i) (Lower semicontinuity):

Suppose $f_{k} \in B V(\Omega)(k=1, \ldots)$ and $f_{k} \rightarrow f$ in $L^{1}(\Omega)$, then

$$
\|D f\|(\Omega) \leq \lim \inf _{k \rightarrow+\infty}\left\|D f_{k}\right\|(\Omega) .
$$

ii) (Compactness):

Let $f_{k} \in B V(\Omega)(k=1, \ldots)$ satisfying $\sup _{k}\left\|f_{k}\right\|_{B V(\Omega)}<+\infty$, then there exist a subsequence $\left(f_{k_{j}}\right)_{1 \leq j \leq+\infty}$ and $f \in B V(\Omega)$ such that

$$
f_{k_{j}} \rightarrow f \text { in } L^{1}(\Omega)
$$

Using the previous lemma, we can prove the following result.

\section{Proposition 7}

i) $\mathcal{U}_{\text {ad }}$ is a nonempty convex closed subset of $B V(\Omega) \cap L^{\infty}(\Omega)$.

ii) Let $\left(h_{n}\right)$ be a sequence of $\mathcal{U}_{a d}$, then there exists $h \in \mathcal{U}_{a d}$ such that $h_{n} \rightarrow h$ in $L^{\lambda}(\Omega)$ (for a subsequence) $\forall \lambda \in[1,+\infty[$.

Proof i) Let $\left(h_{n}\right)_{n \in \mathbb{N}} \subset \mathcal{U}_{a d}$, the assertion (ii) of the Lemma 3 implies that there exists $h \in B V(\Omega)$ such that $h_{n} \rightarrow h$ in $L^{1}(\Omega)$ (for a subsequence). However as $0<a \leq h_{n} \leq b$ a.e in $\Omega$, it follows that $0<a \leq h \leq b$ a.e in $\Omega$. And according to (i) Lemma 3 , we have

$$
\|D h\|(\Omega) \leq \lim \inf _{n \rightarrow+\infty}\left\|D h_{n}\right\|(\Omega) \leq C^{*},
$$

therefore, $h \in \mathcal{U}_{a d}$.

ii) Thanks to the previous assertion, there exists $h \in \mathcal{U}_{a d}$ such that $h_{n} \rightarrow h$ in $L^{1}(\Omega)$ (for a subsequence), in addition

$$
\left\|h_{n}-h\right\|_{L^{\lambda}(\Omega)}^{\lambda} \leq(b-a)^{\lambda-1}\left\|h_{n}-h\right\|_{L^{1}(\Omega)},
$$

for all $\lambda \in[1,+\infty[$, from where the result follows. 


\section{B Some technical lemmas}

Here, we give the general theorem:

Theorem 6 Let $u_{\epsilon}$ be a solution of the following problem

$$
\left\{\begin{array}{l}
u_{\epsilon} \in H^{1}(\Omega) \text { such that } u_{\epsilon}(1)=0, \frac{d u_{\epsilon}}{d x}(0)=0 \text { and } \\
-\frac{d}{d x}\left(\left(a_{\epsilon}(x)\right) \frac{d u_{\epsilon}}{d x}\right)-g_{\epsilon} \frac{d u_{\epsilon}}{d x}=L_{\epsilon}
\end{array}\right.
$$

we suppose that there exist constants $C_{1}, C_{2}, C_{3}$ and $C_{4}$ independent of $\epsilon$ such that

$$
\begin{array}{rr}
0<C_{1} \leq a_{\epsilon}(x) \leq C_{2} \quad \forall x \in[0,1] \\
L_{\epsilon} \in L^{2}(] 0,1[), g_{\epsilon} \in L^{\infty}(] 0,1[) \\
\left\|u_{\epsilon}\right\|_{L^{\infty}(] 0,1[)} \leq C_{3} \quad\left\|L_{\epsilon}\right\|_{L^{2}(] 0,1[)} \leq C_{4} \\
g_{\epsilon} \geq 0
\end{array}
$$

then

$$
\left\|u_{\epsilon}\right\|_{H^{1}(] 0,1[)}+\left\|g_{\epsilon} \frac{d u_{\epsilon}}{d x}\right\|_{L^{1}(] 0,1[)}+\left\|g_{\epsilon} \frac{d u_{\epsilon}}{d x}\right\|_{H^{-1}(] 0,1[)} \leq C .
$$

Let us first prove the following technical lemma in wich $V=\varphi \in H^{1}(] 0,1[), \varphi(1)=0$.

Lemma 4 :

$$
\int_{] 0,1[}\left|g_{\epsilon} \frac{d u_{\epsilon}}{d x}\right| \zeta=\int_{] 0,1[} \operatorname{sign}_{0}\left(\frac{d u_{\epsilon}}{d x}\right)\left(a_{\epsilon} \frac{d u_{\epsilon}}{d x} \frac{d \zeta}{d x}-L_{\epsilon} \zeta\right) \quad \forall \zeta \in V,
$$

where

$$
\operatorname{sign}_{0}(z)=\left\{\begin{array}{cl}
1 & \text { if } z>0 \\
0 & \text { if } z=0 \\
-1 & \text { if } z<0
\end{array}\right.
$$

Proof Let $\operatorname{sign}_{0}^{\sigma}$ be the regularization of the function $\operatorname{sign}_{0}$ such that

$$
\operatorname{sign}_{0}^{\sigma}(z)= \begin{cases}1 & \text { if } z>\sigma \\ \frac{z}{\sigma} & \text { if }-\sigma \leq z \leq \sigma \\ -1 & \text { if } z<-\sigma\end{cases}
$$

with $\sigma>0$. From (42) and the fact that $u_{\epsilon}$ belongs to $V$, we get that $a_{\epsilon} \frac{d u_{\epsilon}}{d x}$ belongs to $H^{1}(] 0,1[)$. It follows that for any $\zeta \in V$, then $\zeta \operatorname{sign}_{0}^{\sigma}\left(a_{\epsilon} \frac{d u_{\epsilon}}{d x}\right)$ also lies in $V$ and can be chosen as a test function in (40). So that

$$
\begin{aligned}
\int_{] 0,1[} g_{\epsilon} \zeta \operatorname{sign}_{0}^{\sigma}\left(a_{\epsilon} \frac{d u_{\epsilon}}{d x}\right) \frac{d u_{\epsilon}}{d x}= & \int_{] 0,1[} \operatorname{sign}_{0}^{\sigma}\left(a_{\epsilon} \frac{d u_{\epsilon}}{d x}\right)\left(a_{\epsilon} \frac{d u_{\epsilon}}{d x} \frac{d \zeta}{d x}-L_{\epsilon} \zeta\right) \\
& +\int_{] 0,1[} a_{\epsilon} \frac{d u_{\epsilon}}{d x}\left(\operatorname{sign}_{0}^{\sigma}\right)^{\prime}\left(a_{\epsilon} \frac{d u_{\epsilon}}{d x}\right) \zeta \frac{d}{d x}\left(a_{\epsilon} \frac{d u_{\epsilon}}{d x}\right)
\end{aligned}
$$

As $\left|\left(\operatorname{sign}_{0}^{\sigma}\right)^{\prime}\left(\frac{d q_{\eta \epsilon}}{d x}\right) \frac{d q_{\eta \epsilon}}{d x}\right| \leq 1$, we get

$$
\begin{aligned}
\int_{] 0,1[} a_{\epsilon} \frac{d u_{\epsilon}}{d x}\left(\operatorname{sign}_{0}^{\sigma}\right)^{\prime}\left(a_{\epsilon} \frac{d u_{\epsilon}}{d x}\right) \zeta \frac{d}{d x}\left(a_{\epsilon} \frac{d u_{\epsilon}}{d x}\right) & \leq C_{2} \int_{\left(\left|\frac{d u_{\epsilon}}{d x}\right| \leq \sigma\right)}\left|\frac{d u_{\epsilon}}{d x}\left(\operatorname{sign}_{0}^{\sigma}\right)^{\prime}\left(\frac{d u_{\epsilon}}{d x}\right)\right|\left|\zeta \frac{d}{d x}\left(a_{\epsilon} \frac{d u_{\epsilon}}{d x}\right)\right| \\
& \leq C_{2} \int_{\left(\left|\frac{d u_{\epsilon}}{d x}\right| \leq \sigma\right)}\left|\zeta \frac{d}{d x}\left(a_{\epsilon} \frac{d u_{\epsilon}}{d x}\right)\right|,
\end{aligned}
$$

however $\int\left(\left|\frac{d u_{\epsilon}}{d x}\right| \leq \sigma\right)\left|\zeta \frac{d}{d x}\left(a_{\epsilon} \frac{d u_{\epsilon}}{d x}\right)\right|$ tends to $\int\left(\frac{d u_{\epsilon}}{d x}=0\right)\left|\zeta \frac{d}{d x}\left(a_{\epsilon} \frac{d u_{\epsilon}}{d x}\right)\right|$ which is equal to 0 (by an application of the Lemma A.4 [27]), then

$$
\int_{] 0,1[} a_{\epsilon} \frac{d u_{\epsilon}}{d x}\left(\operatorname{sign}_{0}^{\sigma}\right)^{\prime}\left(\frac{d u_{\epsilon}}{d x}\right) \zeta \frac{d}{d x}\left(a_{\epsilon} \frac{d u_{\epsilon}}{d x}\right) \rightarrow 0 .
$$

Then passing to the limit on $\sigma$ in (46) and using (44), we obtain the result of the lemma. 
Proof of theorem 6

Let us define $\lambda_{\epsilon}=g_{\epsilon} \frac{d u_{\epsilon}}{d x}$

$$
\gamma_{\epsilon}=\frac{1}{1+\left\|\lambda_{\epsilon}\right\|_{L^{1}(] 0,1[)}+\left\|\lambda_{\epsilon}\right\|_{H^{-1}(] 0,1[)}}, \widetilde{u}_{\epsilon}=\gamma_{\epsilon} u_{\epsilon}, \tilde{\lambda}_{\epsilon}=\gamma_{\epsilon} \lambda_{\epsilon},
$$

then

and from 40

$$
\gamma_{\epsilon}+\left\|\widetilde{\lambda}_{\epsilon}\right\|_{L^{1}(] 0,1[)}+\left\|\widetilde{\lambda}_{\epsilon}\right\|_{H^{-1}(] 0,1[)}=1
$$

$$
-\frac{d}{d x}\left(a_{\epsilon} \frac{d \widetilde{u}_{\epsilon}}{d x}\right)=\widetilde{\lambda}_{\epsilon}+\gamma_{\epsilon} L_{\epsilon}
$$

Taking $\widetilde{u}_{\epsilon}$ as a test function in (48), we get

$$
\begin{aligned}
\int_{] 0,1[} a_{\epsilon}\left(\frac{d \widetilde{u}_{\epsilon}}{d x}\right)^{2} & =\int_{] 0,1[} \widetilde{\lambda}_{\epsilon} \widetilde{u}_{\epsilon}+\int_{] 0,1[} \gamma_{\epsilon} L_{\epsilon} \widetilde{u}_{\epsilon} \\
& =\gamma_{\epsilon} \int_{] 0,1[}\left(\widetilde{\lambda}_{\epsilon}+\gamma_{\epsilon} L_{\epsilon}\right) u_{\epsilon},
\end{aligned}
$$

From (43), $u_{\epsilon}$ is bounded in $L^{\infty}(] 0,1[)$. Moreover $\widetilde{\lambda}_{\epsilon}+\gamma_{\epsilon} L_{\epsilon}$ is bounded in $L^{1}$ (]0,1[) from (47) and (43), using (41), we get:

$$
\left\|\widetilde{u}_{\epsilon}\right\|_{H^{1}(] 0,1[)}^{2} \leq C \gamma_{\epsilon} .
$$

Now choosing $\phi \in H_{0}^{1}(] 0,1[)$ as a test function in (48), we get

$$
\int_{] 0,1[} a_{\epsilon} \frac{d \widetilde{u}_{\epsilon}}{d x} \frac{d \phi}{d x}=\int_{] 0,1[} \widetilde{\lambda}_{\epsilon} \phi+\int_{] 0,1[} \gamma_{\epsilon} L_{\epsilon} \phi
$$

which is rewritten as

$$
\int_{] 0,1[} \widetilde{\lambda}_{\epsilon} \phi=\int_{] 0,1[} a_{\epsilon} \frac{d \widetilde{u}_{\epsilon}}{d x} \frac{d \phi}{d x}-\int_{] 0,1[} \gamma_{\epsilon} L_{\epsilon} \phi
$$

so that

$$
\left|\int \widetilde{\lambda}_{\epsilon} \phi\right| \leq C_{2}\left\|\widetilde{u}_{\epsilon}\right\|_{H^{1}(] 0,1[)}\|\phi\|_{H_{0}^{1}(] 0,1[)}+\gamma_{\epsilon}\left\|L_{\epsilon}\right\|_{L^{2}(] 0,1[)}\|\phi\|_{H_{0}^{1}(] 0,1[)} .
$$

From (43) we get

$$
\left\|\widetilde{\lambda}_{\epsilon}\right\|_{H^{-1}(] 0,1[)} \leq C\left(\gamma_{\epsilon}+\left\|\widetilde{u}_{\epsilon}\right\|_{H^{1}(] 0,1[)}\right) .
$$

Now, let us suppose

$$
\text { Assumption }(\mathrm{H}) \quad \lambda_{\epsilon} \text { is not bounded in } L^{1}(] 0,1[) \cup H^{-1}(] 0,1[)
$$

Then $\gamma_{\epsilon}$ tends (for a subsequence) to 0 , then $\widetilde{u}_{\epsilon}$ and $\widetilde{\lambda}_{\epsilon}$ converge to 0 respectively in $H^{1}(] 0,1[)$ and $H^{-1}(] 0,1[)$ and according to (47)

$$
\left\|\widetilde{\lambda}_{\epsilon}\right\|_{L^{1}(] 0,1[)} \rightarrow 1
$$

We aim to compute $\left\|\widetilde{\lambda}_{\epsilon}\right\|_{L^{1}(] 0,1[)}$ by way of the characterization

$$
\left\|\tilde{\lambda}_{\epsilon}\right\|_{L^{1}(] 0,1[)}=\sup _{w \in \mathbb{B}(0,1)} \int_{0}^{1}\left|\tilde{\lambda}_{\epsilon}\right| w d x
$$


in which $\mathbb{B}(0,1)$ denotes the unit ball of $C_{0}([0,1])$.

Let us choose $\phi$ in $H^{1}(] 0,1[)$ such that $\phi \geq 1$ so that for any $w$ in $\mathbb{B}(0,1)$

$$
w \leq \phi
$$

Such a function $\phi$ cannot belong to $V$ as it cannot be 0 for $x=1$. So we locally modify $\phi$ by introducing for any small non negative parameter $\sigma$

$$
v_{\sigma}(z)=\left\{\begin{array}{cl}
\sigma & \text { if } z \leq 1-\sigma, \\
\frac{\phi(1)-\sigma}{\sigma} z+\frac{\phi(1)(\sigma-1)+\sigma}{\sigma} & \text { if } z \geq 1-\sigma,
\end{array}\right.
$$

Let us write from $(53)$

$$
\int_{0}^{1}\left|\widetilde{\lambda}_{\epsilon}\right| w \leq \int_{0}^{1}\left|\tilde{\lambda}_{\epsilon}\right| \phi=\int_{0}^{1}\left|\tilde{\lambda}_{\epsilon}\right|\left(\phi-v_{\sigma}\right)+\int_{0}^{1}\left|\tilde{\lambda}_{\epsilon}\right| v_{\sigma}
$$

we can use $\zeta=\gamma_{\epsilon}\left(\phi-v_{\sigma}\right)$ as a test function in 4 so that (54) leads to:

$$
\begin{aligned}
\int_{0}^{1}\left|\widetilde{\lambda}_{\epsilon}\right| w & \leq \int_{0}^{1} \operatorname{sign}_{0}\left(\frac{d \widetilde{u}_{\epsilon}}{d x}\right) a_{\epsilon} \frac{d \widetilde{u}_{\epsilon}}{d x} \frac{d \phi}{d x}-\int_{0}^{1} \operatorname{sign}_{0}\left(\frac{d \widetilde{u}_{\epsilon}}{d x}\right) a_{\epsilon} \frac{d \widetilde{u}_{\epsilon}}{d x} \frac{d v_{\sigma}}{d x} \\
& -\gamma_{\epsilon} \int_{0}^{1} \operatorname{sign}_{0}\left(\frac{d \widetilde{u}_{\epsilon}}{d x}\right) L_{\epsilon}\left(\phi-v_{\sigma}\right)+\int_{0}^{1}\left|\widetilde{\lambda}_{\epsilon}\right| v_{\sigma}
\end{aligned}
$$

which is rewriten as

$$
\int_{0}^{1}\left|\widetilde{\lambda}_{\epsilon}\right| w \leq I_{1}-I_{2}-I_{3}+I_{4}
$$

From (41) and (49): $\quad\left|I_{1}\right| \leq C \sqrt{\gamma_{\epsilon}}\left\|\frac{d \phi}{d x}\right\|_{L^{2}(] 0,1[)}$

From (41) and the definition of $v_{\sigma}: \quad I_{2} \geq 0$

From (43) and the definition of $v_{\sigma}: \quad I_{3} \geq-C \gamma_{\epsilon}\left(1+\left\|v_{\sigma}\right\|_{L^{2}(] 0,1[)}\right)$

As $\tilde{\lambda}_{\epsilon}$ belongs to $L^{\infty}(] 0,1[)$ from (42),(43) and $v_{\sigma} \geq 0$ then:

$$
I_{4} \leq\left\|\widetilde{\lambda}_{\epsilon}\right\|_{L^{\infty}(] 0,1[}\left\|v_{\sigma}\right\|_{L^{1}(] 0,1[)}
$$

As constant $C$ does not depend on $\sigma, w$ or $\epsilon$, we can first let $\sigma$ tend to zero so that $\left\|v_{\sigma}\right\|_{L^{2}(] 0,1[)} \rightarrow 0$ and we get

$$
\int_{0}^{1}\left|\widetilde{\lambda}_{\epsilon}\right| w \leq C \sqrt{\gamma_{\epsilon}}\left\|\frac{d \phi}{d x}\right\|_{L^{2}(] 0,1[)}+C \gamma_{\epsilon}
$$

as $\phi$ does not depend on $w$ and as $\gamma_{\epsilon}$ tends to zero, we get

$$
\left\|\widetilde{\lambda}_{\epsilon}\right\|_{L^{1}(] 0,1[} \leq C \sqrt{\gamma_{\epsilon}}
$$

which is impossible from (51) and the assumption $(\mathrm{H})$.

So assumption $(\mathrm{H})$ is not true and this implies that there exists a constant $C>0$ such that

$$
\left\|\widetilde{\lambda}_{\epsilon}\right\|_{L^{1}(] 0,1[}+\left\|\widetilde{\lambda}_{\epsilon}\right\|_{H^{-1}(] 0,1[} \leq C
$$

According to (49) and the definition of $\widetilde{u}_{\epsilon}$, we have

$$
\left\|u_{\epsilon}\right\|_{\left.\left.H^{1}(] 0,1\right]\right)}^{2} \leq \frac{C}{\gamma_{\epsilon}}=C\left(1+\left\|\widetilde{\lambda}_{\epsilon}\right\|_{L^{1}(] 0,1[}+\left\|\widetilde{\lambda}_{\epsilon}\right\|_{H^{-1}(] 0,1[}\right)
$$

Then from (56) we get

$$
\left\|u_{\epsilon}\right\|_{\left.\left.H^{1}(] 0,1\right]\right)} \leq C
$$

so estimate (45) is obtained from (56) and (58). 